\title{
Spark detection and search for high-voltage Paschen leaks in a large superconducting coil system
}

Juergen Baldzuhn, Hans Stephan Bosch, Kirk Gallowski, Heinz Grote, Thomas Klinger, Christian Kopplin, Michael Nagel, Kerstin Rummel, Thomas Rummel, Konrad Risse, Felix Schauer, Olaf Volzke, Lutz Wegener, Andreas Werner Max-Planck Institut für Plasmaphysik IPP

Wendelsteinstr. 1, 17491 Greifswald, Germany

Michael Weise

Forschungs- und Transferzentrum Leipzig e.V.

Karl-Liebknecht-Str. 143, 04277 Leipzig, Germany

\begin{abstract}
The plasma fusion experiment Wendelstein 7-X (W7-X) uses a system of 50 non-planar and 20 planar superconducting coils. These coils produce the magnetic field that is required to confine the plasma. Magnetic flux densities up to $3 \mathrm{~T}$ can be reached in the center of the plasma. Supercritical helium is used to cool down the coils to operating temperatures below $4 \mathrm{~K}$. The coils are specified for currents up to $18 \mathrm{kA}$. A critical issue in all superconductors is the occurrence of quenches. These are unwanted local transitions from superconductivity to normal conductivity. If that happens, the coil current has to be discharged as fast as possible into a dump resistor. However, the strong current change will produce a self-induced high voltage with values up to several $\mathrm{kV}$. Therefore, the electrical coil insulation versus ground has to be high-voltage proof to avoid a high-current arc in any circumstance. Worst-case scenario is a quench, that is induced by a loss of thermal insulation after a leak in the helium supply lines, because then Paschen-minimum conditions could be given. This paper describes some highvoltage test procedures and techniques employed to test and qualify the coil system against that scenario. Some of these techniques are used today for the routine coil tests. Some other turned out as inefficient for daily use. Essential for a safe machine operation is the detection of insulation defects, but also on their localization along the superconductor, for the sake of a later repair.
\end{abstract}

Keywords - superconductivity, Paschen-conditions, high-voltage testing

\section{Introduction, motivation}

Since the year 2015, the plasma fusion experiment Wendelstein 7-X (W7-X) [1] is in operation [2]. It provides fusion relevant plasma discharges to exploit the potential of a magnetically confined toroidal plasma facility as a clean and reliable source of nuclear power for the future. W7-X is of the so-called stellarator-type. It needs no internal plasma current to accomplish the magnetic plasma confinement, as this is the case in the experiments of the tokamak-type. The first reactor-scale tokamak ITER, (International Test Experimental Reactor) [3] is presently build up in Cadarache, France. ITER will provide one day about $500 \mathrm{MW}$ of fusion power.

All large fusion plasma experiments employ superconducting coil systems to produce the magnetic field for the confinement of the plasma. This is essential to sustain a steady-state magnetic field in the range of about 5T (for a reactor) inside the plasma. Thus, steady-state plasma operation is possible without the need of electrical power for a normal-conducting coil system.

Fig. 1 shows a sketch of the W7-X stellarator arrangement. The 70 superconducting coils in W7-X are connected in seven series of 10 coils each [4]. Currents up to $18 \mathrm{kA}$ can flow in each series. The coils are situated inside an evacuated vessel, in order to minimize the heat conductivity of the residual gas. A closed radiation shield, made of brass and copper, in combination with a multi-layer thin foil insulation, reduce the heat flow further. Access to the coils is possible only at the electrical coil connection-ends and at the quench detection wires. The metallic vacuum vessel and radiation shields act as an almost perfect Faraday's screen. Optical windows do not exist, because they are a potential source of thermal radiation onto the cryogenic installations. The entire torus consists of five almost identical modules, each of them spreading over $360^{\circ} / 5=72^{\circ}$ around the torus. 


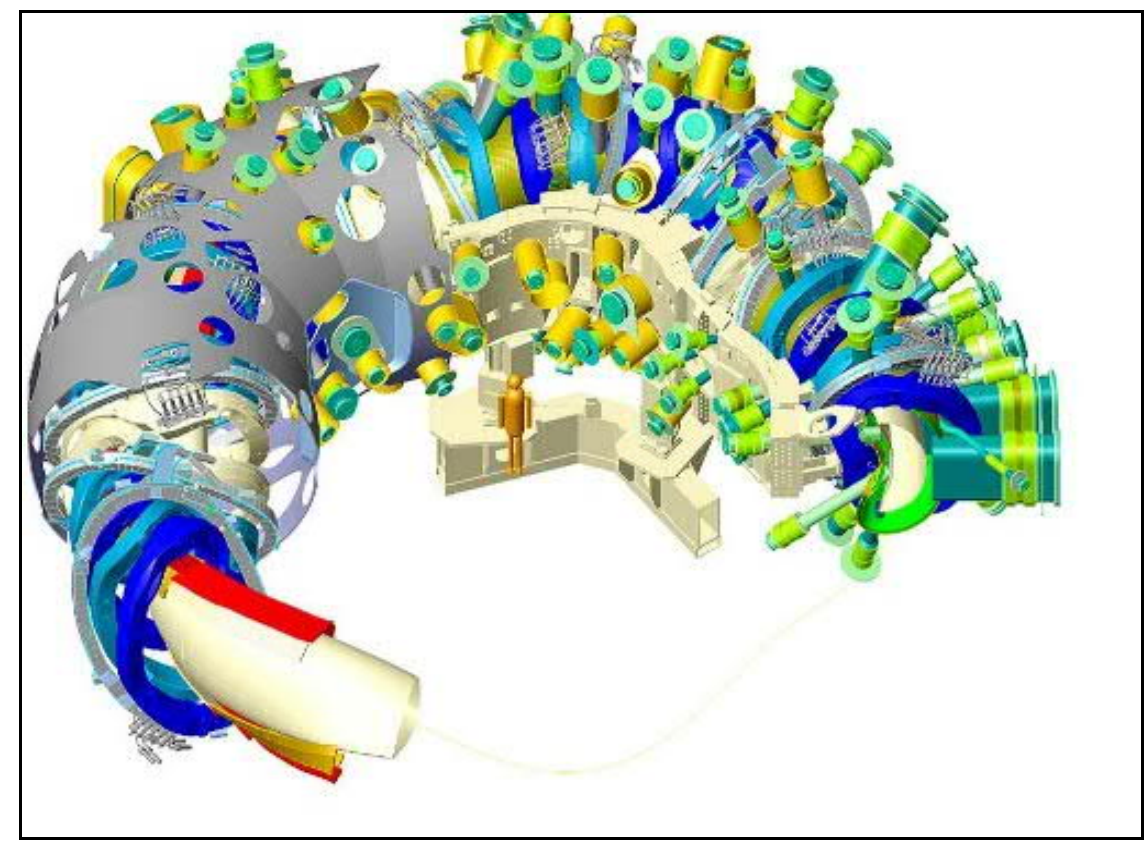

Fig. 1: Sketch of the W7-X hardware and coil arrangement. Shown is the plasma, a part of the plasma vessel, the coil set, the ports and the cryostat skin together with a part of the coil support structure.

In addition, each coil winding is situated inside a closed stainless steel casing with a wall thickness of 3-4 cm. This casing has to carry the mechanical forces, mainly Lorentz forces, in the range of several $10 \mathrm{kN} / \mathrm{m}^{2}$. This mechanical support is essential, because it sustains the complex shape and geometry of the coils during current operation. It stabilizes the coils also during cool-down and warming-up, where the overall size of the coils changes up to about $15 \mathrm{~mm}$.

In W7-X, the Cable-In-Conduit-Conductor (CICC) technique is used. Filaments of a Niobium-Titanium alloy are embedded in a copper matrix. The copper wires lie loosely in a hollow aluminum cable, which is surrounded by the flow of the coolant helium. Supercritical helium is used at a nominal operation pressure of about 6 bar. All coil windings, and all cables that interconnect the coils inside the cryostat, are electrically insulated by handmade expoxy sleeves. Great care was taken during the coil production to guarantee vacuum tightness of that insulation. Thus, it should be assured, that no electrically conducting channels remain between the metallic cables and the surrounding vacuum. This condition is essential for the case of a quench. During such a quench, the superconductor experiences a local loss of super-conductivity. As a result, a considerable amount of Ohmic heat in the coil will warm up the cable within a few seconds to temperatures, where the thermal stresses inside the structure can damage the entire coil irreversibly.

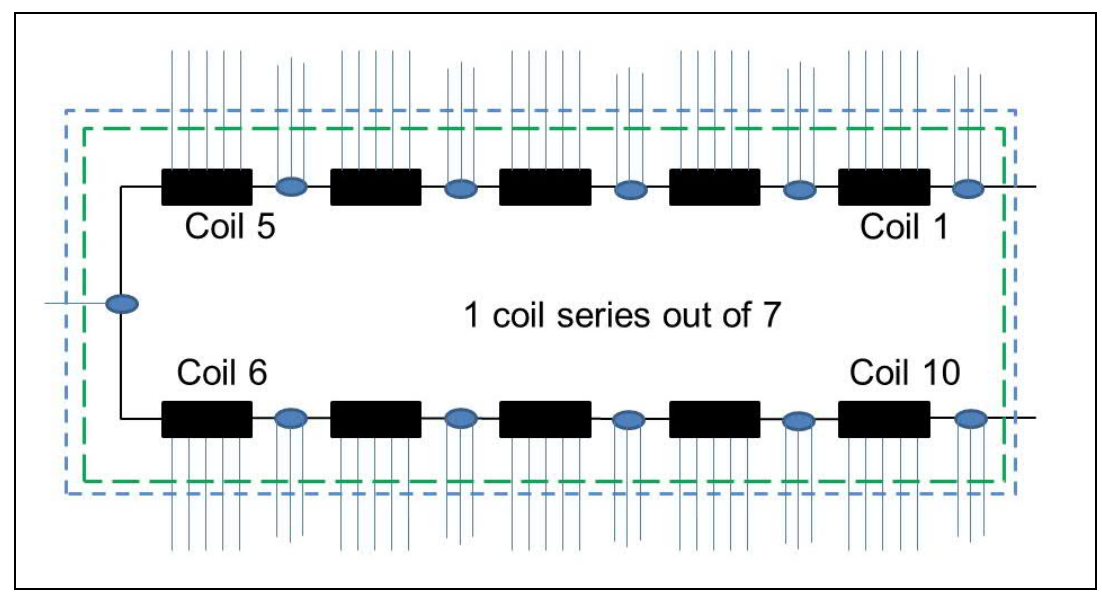

Fig. 2: Sketch of one coil series out of seven in W7-X. Shown are the ten superconductor coils (black) with the connecting normal conductive joints (ellipses) and the quench-detection wires going away from the coils. Symbolically, the radiation shield and the cryostat vessel wall are indicated, too (dashed lines). 
In W7-X, a quench-detection system is installed. This system measures and surveys the Ohmic voltage drop along the cables within the coils. If this voltage violates an upper threshold voltage for more than typically about $50 \mathrm{~ms}$, then a "quench-alarm" is triggered. In such a case, the coil current has to be dumped as quickly as possible into dump resistors, in order to avoid the above-described damages. Each coil has 18 individual quenchdetection wires that reach through the thermal and electrical coil insulation. In total, more than 1200 of these wires are installed in W7-X. Each wire has a typical length of several $10 \mathrm{~m}$. Fig. 2 shows one series of superconducting coils together with the quench-detection wires, which are reaching through the radiation shielding and the cryostat vessel wall.

This arrangement makes the W7-X superconducting system a complex 3D network with considerably large inductances (the entire coil system has $1 \mathrm{H}$ ), numerous stray capacities (each coil winding has about $10 \mathrm{nF}$ ) and a combination of superconducting or normal conducting cables.

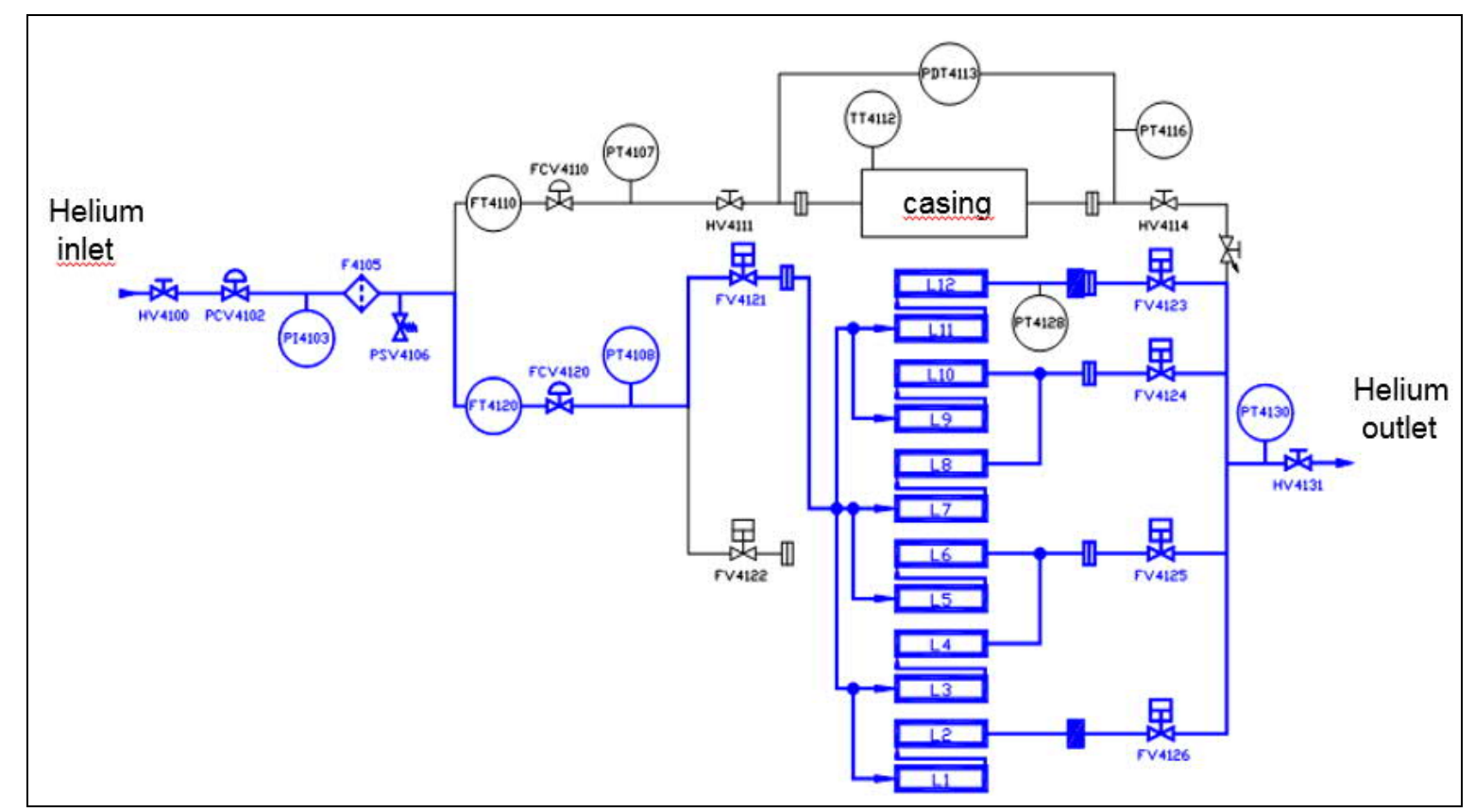

Fig. 3: Sketch of the hydraulic pipes and superconductor windings of one non-planar coil, together with some of the sensors. The windings L1-L12 are superconducting, in principle the entire arrangement shown here is under high-voltage during tests or a fast current discharge. The casing is the stainless steel coil casing, the cooling helium flows from the inlet to the outlet. The different types of valves are labelled by HV, PCV, FVC, FV. Pressure gauges are labelled by PI, temperature gauges by PT or TT, flow meters by FT, the pressure difference gauge by PDT. The blue lines include the coil pipes, the black lines the coil casing system. Colours are visible only in the online version of the paper.

Fig. 3 shows a rough sketch of the helium cooling pipes, i.e. the hydraulic scheme of one individual coil, together with some of the sensors for pressure, temperature and coolant flow and the internal series of the windings. During high voltage tests, or if high voltage is applied on the coil windings, some of these sensors are also exposed to HV. Therefore, they are insulated carefully and individually, versus ground as well as versus the surrounding vacuum. Many more external gauges exist, (for instance the strain gauges) but these are not shown here. The superconductor windings (L1 - L12) are separate from the coil casing which has to stabilize the coil shape against several tons of Lorentz forces during operation (labelled as casing). During high-voltage testing, the helium pipes have to remain under high pressure (min. 6 bar) to avoid Paschen minimum discharges inside the pipes. 


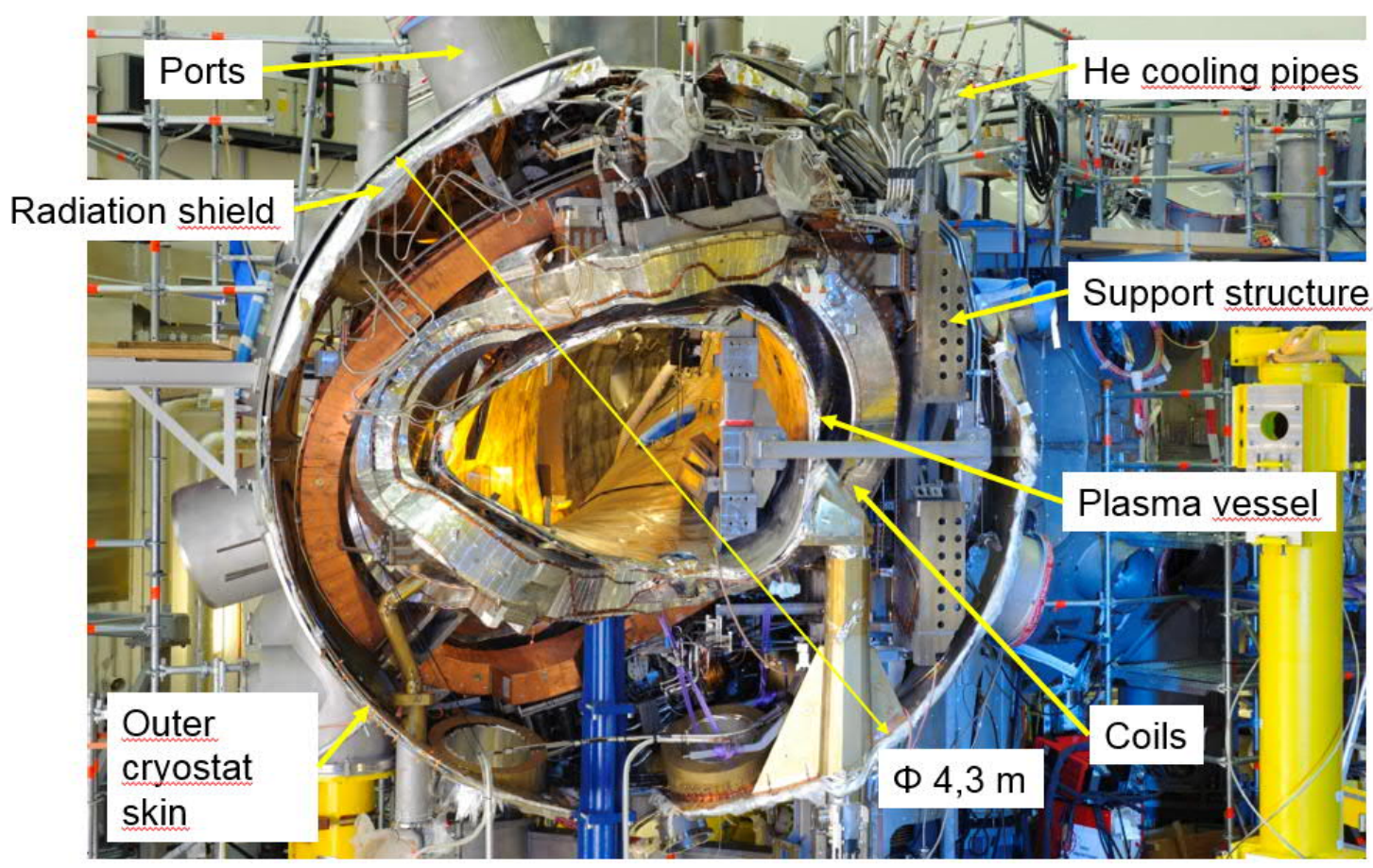

Fig. 4: Photograph of a small section of W7-X, taken during assembly. Today, all components are covered by steel and are invisible. The triangular plasma vessel is surrounded by the superconducting coils, the latter are held in position by the support structure. Some of the helium cooling pipes can be seen on top of the vessel, within the cryostat skin (made of steel with a thickness $\approx 20-30 \mathrm{~mm}$ ) sits the cooled radiation shield. The cross section shown here has a diameter of $4.3 \mathrm{~m}$.

Fig. 4 shows, just for illustration of the size and complexity of the system, a small fraction of the coil arrangement for W7-X, as this was visible during the assembly phase. Some of the major components are labelled in the figure. One can see one non-planar and one planar coil (out of 70), some of the sensor cablings and helium cooling pipes. Inside the coils sits the plasma vessel with a diameter of about $1.5 \mathrm{~m}$, here visible as a triangular shape. The radiation shield is a closed structure, being cooled to about $30-70 \mathrm{~K}$ during operation. The number of gaps or holes within the latter has to be minimized to minimize radiation loads to the coils. On the other hand, the access is strongly hindered by that shield. Possible sparks or discharges inside the coil arrangement during tests are not visible with this present set-up, therefore electronic methods have to be developed as described below.

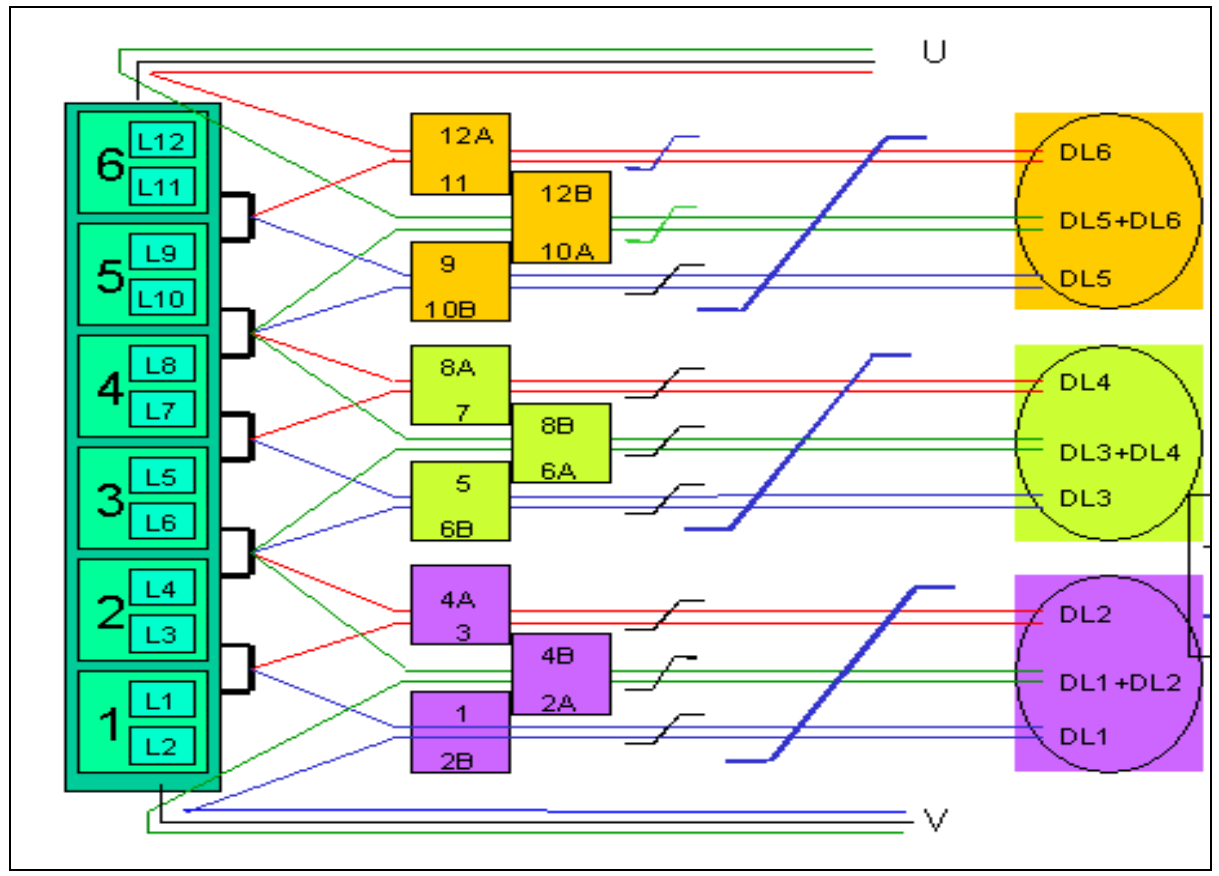


Fig. 5: Part of the qd-wiring scheme of one individual coil. One the left side are the six double windings (L1-L12) of the superconductor, 24 qd-cables are shown. Several qd-wires are connected to the same points on the superconductor in order to provide redundancy for the case of cable breaks.

Fig. 5 shows a part of the quench detection (qd) wiring of one individual coil. The qd-wires are directly soldered to the superconductor on their cold end; the warm end reaches through the cryostat skin to external qdelectronics. There, very sensitive Wheatstone bridge amplifiers detect permanently the Ohmic voltage drop along the superconducting cable, in case a voltage threshold is violated for some time, a fast current discharge is initiated, i.e. the superconductor current is dumped in the dump resistors. Details follow below. The qd-cable arrangement and its insulation are essential for the operation of the machine, because a quench can destroy W7$\mathrm{X}$. Particular care for insulation is required, because the entire qd-wiring will be on high-voltage during tests or a fast current discharge.

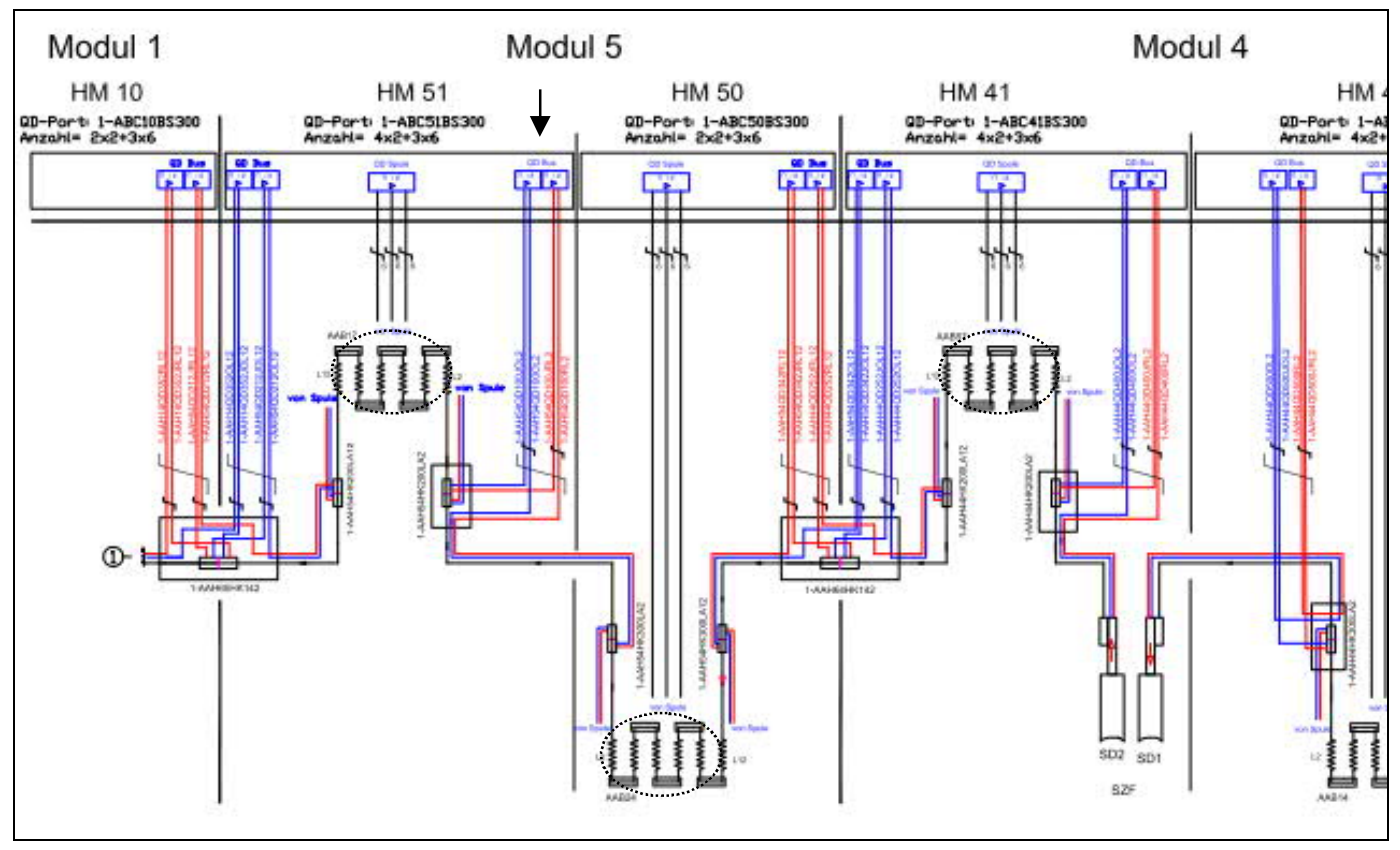

Fig. 6: Cutout of a sketch of a series of 10 individual coils (within the dotted black ellipses) together with some of the connecting joints (between the coils), and some of the qd-wires for the external bus-bar cabling (horizontal lines, partly super-conducting, partly normal conducting). We show only 3 out of 10 coils for better visibility of the details. The coils are situated within the modules $1-5$ of W7-X. We show here only the modules 1, 4 and 5. Some of the bus-bar qd-wires are also shown (blue and red vertical lines). The arrow refers to the position, where one of the four oscilloscope input channels was connected to the qd-wires. This was done as an attempt to localize a weak insulation point. Refer here to the fig. 20 below.

Fig. 6 shows a series of ten coils situated in the modules $1-5$ of W7-X. Each coil is represented by the six double windings L1 - L12 (within the dotted circles), being interconnected by the superconducting bus-bar system. As this bus-bar system requires a qd-wiring, as well, these are shown here. The connecting joints (boxes between the coils) are normal-conducting, but require cooling to about $4 \mathrm{~K}$ as well to minimize the Ohmic heat production at that point. During high-voltage tests or a fast current discharge, this entire arrangement is under high-voltage and is therefore relevant for all considerations mentioned in this paper. The superconducting system of W7-X consists in total of seven of these arrangements, being distributed over the five machine modules.

The dump resistors for the non-planar coils have a value of $74 \mathrm{mOhm}$ and guarantee that the currents can be dumped with a halftime of roughly 5 seconds. Due to the fast current decay and the high impedance of the coil system (about 1 Henry), a high voltage is induced on the superconductor. The maximum value of this high voltage remains always below $950 \mathrm{~V}$. Finite-element calculations [5] for the superconductor predict a maximum temperature increase at the quench hot-spot of less than $130 \mathrm{~K}$. These values are considered as uncritical for the coils. 


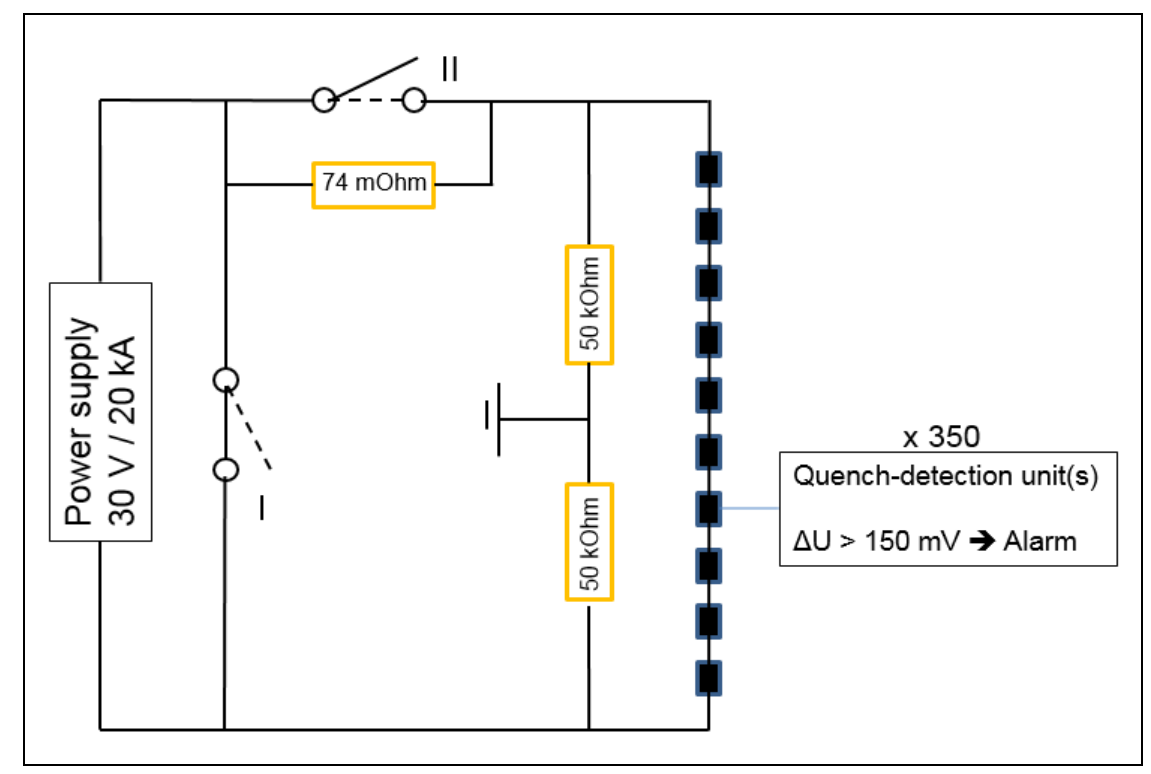

Fig. 7: Very rough, principal sketch of one coil series (one out of seven, the vertical line of black boxes) together with the dump resistor (on top, $74 \mathrm{mOhm}$ ) and two switches labelled as I and II. One out of 350 quench detection units is shown symbolically on the right. Two resistors in series $(50 \mathrm{kOhm}$ each) reduce the maximum potential at the tail-ends of the coils versus ground by one half. The coil power supply (left side) can provide a max. current of $20 \mathrm{kA}$ at $30 \mathrm{~V}$ input voltage. During normal operation the switches are open (switch I, dashed line) resp. closed (switch II, dashed line). If the coil current is dumped into the dump resistor, switch I is closed (solid line) while switch II is open (solid line). The time sequence is described in the text.

Fig. 7 shows a rough sketch of one series made of ten coils, together with the high current switches, labelled I and II, and one quench-detection unit out of 350. In order to minimize the high-voltage in case of a quench, two resistors with $50 \mathrm{kOhm}$ each divide the voltage between the coil tail-ends into two identical halves. The grounding at the symmetry point between them guarantees the ground potential at this location. Hence, the maximum voltage of each component, being connected to the coils, versus ground is limited to $1.9 \mathrm{kV} / 2=950$ V. During normal operation, the power supply is connected to the series of coils, while switch I is open and switch II is closed. Consider, that the power supply needs to provide a voltage only for the case that a current $\operatorname{ramp} d I(t) / d t$ has to be driven in the coils, and for the very small voltage drop in the normal-conducting joints between the coils. If a quench is detected (i.e. if the Ohmic voltage drop between two connection points inside one coil increases above about $150 \mathrm{mV}$ ), the quench detection units provide an alarm signal. This alarm signal induces a regulation of the output of the power supply immediately to $0 \mathrm{~V}$ at the outlet. With a delay of about 37 $-50 \mathrm{~ms}$, switch I is closed to protect the power supply against the high voltage. After a further delay between about $300-350 \mathrm{~ms}$, switch II opens in order to activate the dump resistor. Now, the Ohmic resistivity can absorb the energy, which is stored in the magnetic field of the coil system.

The high complexity of the coil system, together with the poor accessibility, makes high-voltage tests essential. This holds in particular for pressure values close to the Paschen-minimum, because then the probability for an arc are maximum.

I. Tests prior to the installation into $\mathrm{W} 7-\mathrm{X}$

Prior to installation into the W7-X cryostat, each of the 70 coils had been tested individually for correct internal wiring of the windings [6], correct function of all attached gauges and sensors, and attainment of superconductivity at the predicted transition temperature. Essential was the test for sufficient high-voltage insulation of the coil insulation, even at vacuum pressure values in vicinity to the Paschen-minimum (the socalled Paschen-tightness) [7]. This initial phase was essential for testing of the coils, but also for a learning procedure how to handle the coils, perform and assess the tests and conduct the safe operation. In addition, control and steering of the manufacture of the coils turned out as essential in the very process of fabrication.

These tests were done in two cryogenic vacuum vessels at the Commissariat a l'Energie Atomique CEA, Saclay, France in the years 2003 - 2013. In some coils, a quench was provoked in order to investigate the resulting thermal, high-voltage and mechanical stresses during such an event, and to test the procedure of the rapid current dump. One major task of the coil tests was the effort to make the coils Paschen-tight as long as access to them was possible. We took advantage of the fact that the coil production was still ongoing. Therefore, all required spare parts, machinery and skilled personal staff had been available for re-work and repair.

The worst failure-case is a combination of a leak in the helium supply lines, with the consequence of a quench because of the missing thermal insulation of the coils. The insulation will be lost in this case, because for gas 
pressures $>10^{-4} \mathrm{hPa}$ an enhanced heat conduction will be induced. Therefore, the required cryogenic temperatures in the coils cannot be maintained any more. The high-voltage peak will then occur simultaneously with background pressure conditions close to the Paschen-minimum. A spark, followed by an arc might then be ignited, driven by the high superconductor current.

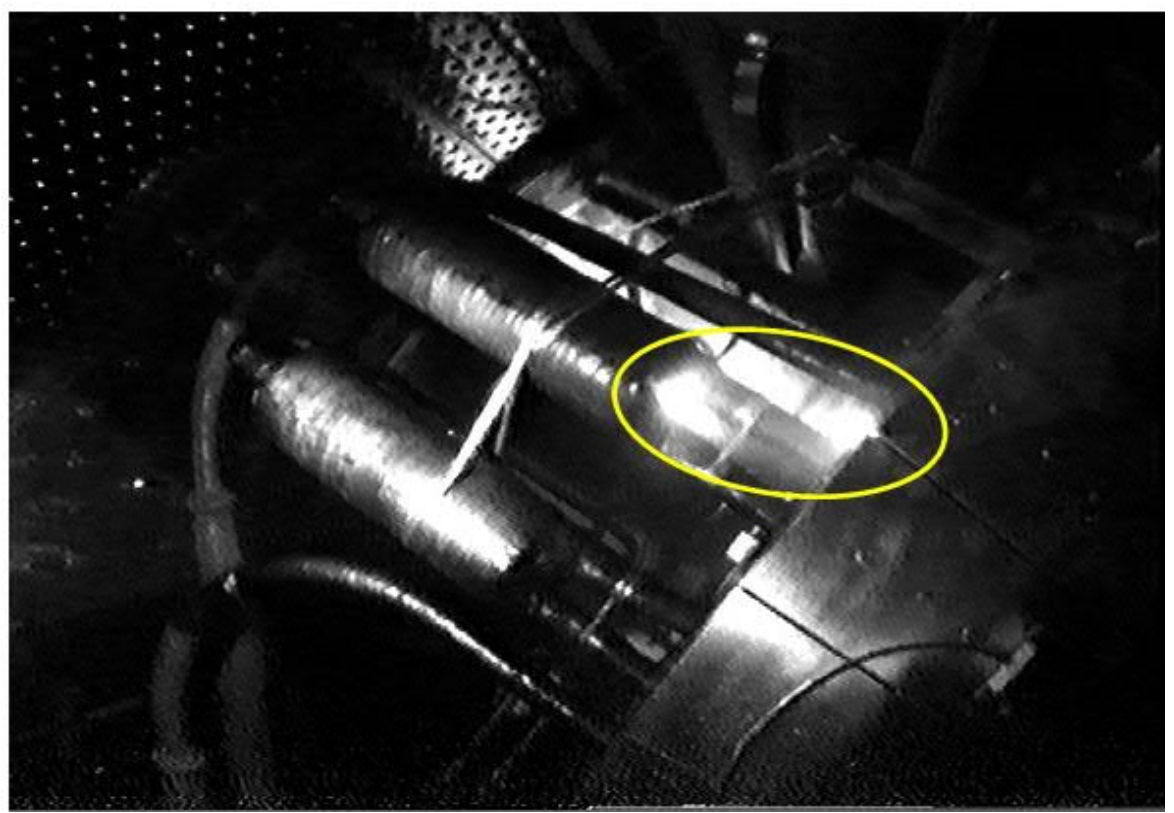

Fig. 8: Video photograph taken during the coil tests in Saclay. A discharge occurs between a superconducting joint of the coil (left hand side) and the grounded coil casing (right hand side); at a background pressure of $1 \mathrm{hPa}$ and a test voltage of $6 \mathrm{kV}$ (the discharge is marked by the ellipse).

For helium, these conditions are fulfilled at a voltage of about only $200 \mathrm{~V}$ (which is exceeded by the induced high voltage) and a distance-pressure product of about 10-100 $\mathrm{mm} \times \mathrm{hPa}$. Due to the complexity of the W7-X cryostat, the potential discharge distance varies between $1 \mathrm{~mm}$ and $20 \mathrm{~m}$. Hence, a large pressure interval exists, within which a spark could be ignited, if the electrical coil insulation is not perfect. During high voltage tests at the beginning of the W7-X construction phase, we observed sparks in the pressure range between $0.01 \mathrm{hPa}$ up to $50 \mathrm{hPa}$ on coils with imperfect electrical insulation.

Such a worst failure-case occurred during the coil tests in Saclay, and it destroyed a part of the test facility. Our assumption that the magnetic field surrounding the coil might blow out any arc was not fulfilled. Instead, the initial spark formed an arc, which welded several holes into gas pipes inside the vessel (within 1-2 s) and destroyed parts of the electrical connections. Finally, the inflowing gas quenched the arc. Fortunately, all damages could be repaired quickly.

Another large Paschen-test tank with a diameter of $5 \mathrm{~m}$ is available in our institute in Greifswald. It is large enough to house one superconducting coil. After delivery, each coil had been tested once again inside this tank, however no cryogenic cooling could be provided. This tank can be evacuated, and the installations allow for HV and Paschen-tests at variable pressures and testing conditions. That tank helped for testing the coils after the transportation immediately before installation, and supported us strongly in learning and assessing the present and future high-voltage tests.

As each HV test will stress the coil insulation, the total number of tests, as well as the test voltage, are today reduced to a minimum. Numerous testing concepts had been tried in advance in Saylay, and a few of them are today implemented on W7-X. Focus was always on test strategies, which stress the coils as little as possible. These are described in the next chapters.

\section{Test procedures on $\mathrm{W} 7-\mathrm{X}$}

High-voltage and Paschen-tests are performed today on a regular and periodic basis. A first long series of tests was performed during the assembly phase of W7-X during the years 2012-2016. The focus was here on the correct cabling of the coils, safe and well insulated stowing of all superconductor and sensor cables inside the cryostat, local tests of Paschen-tightness of the cable insulation and the connectors, and local vacuum leak tests of the feed-throughs.

Since plasma operation in W7-X was started in the year 2015, the test procedure is continued in parallel to the plasma operation. All tests are organized according to a strict and well-documented testing plan. They are 
performed under warm and cryogenic conditions, under air pressure and vacuum, i.e. between $10^{-4} \mathrm{hPa}$ at room temperature down to $10^{-8} \mathrm{hPa}$ under cryogenic temperatures. In addition, tests are done under vacuum conditions in vicinity to the minimum of the Paschen-curve for helium. For these tests, the pressure values are stabilized in steps between $20 \mathrm{hPa}, 2 \mathrm{hPa}$ and $0.2 \mathrm{hPa}$. For each pressure value, the high voltage is applied for 1 minute to sustain flat-top conditions. Typical test voltages are $2.5 \mathrm{kV}$ for the non-planar and $1.6 \mathrm{kV}$ for the planar coils. The maximum test current is always limited by the test power supply to values below $100 \mu \mathrm{A}$.

No sparks or current peaks are allowed during the entire testing period. The maximum test current must be below $2.5 \mu \mathrm{A}$. For the high-voltage tests under air pressure or good vacuum below $10^{-3} \mathrm{hPa}$, the maximum test current has to be smaller than $10 \mu \mathrm{A}$. The helium pressure inside the supply tubes is always above 2.5 bar to avoid discharges inside the pipes. The Paschen-tests can be conducted only at ambient temperature, because under cryogenic temperatures the heat conductivity from the coils to the vessel would be too high for the required testing pressures.

One aspect of the tests is the indication of Paschen-tightness of the entire insulation. Another is the localization of possible weak points in the insulation. This is illustrated by fig. 8, showing a video photograph of a Paschentest performed at CEA, Saclay. It shows a discharge between the superconductor and the housing of one coil at a background pressure of $1 \mathrm{hPa}$. Thanks to the video technique, the origin of the spark could easily be identified and repaired straightforward. Due to the missing access to the coils in W7-X, however, this video technique cannot be applied any more today.

Another issue that had to be addressed was the shape of the Electro-Magnetic Pulse (EMP), which can be measured with an antenna arrangement. These antennas could be installed permanently in W7-X. Prior to the installation in W7-X, the EMP characteristics of possible sparks had to be investigated in the lab. This is described now.

A spark gap (0.5 mm width with needle electrodes) was placed in a vacuum tank (see the set-up of the vacuum test vessel below) and sparks were ignited. The EMP was recorded (if it appeared) after measurement with a 0.2 $\mathrm{m}$ long rod antenna, while the vacuum pressure in the vacuum vessel was varied over a wide range. The working gas was air. The typical spark energy was in the range of $<1 \mu \mathrm{J}$. In parallel, the average electrical current was measured. The maximum current was limited to values $<100 \mu \mathrm{A}$. The table I shows the measurement results.

\begin{tabular}{|c|c|c|}
\hline $\mathrm{P}[\mathrm{hPa}]$ & $\mathrm{U}[\mathrm{V}]$ & $\mathrm{I}[\mu \mathrm{A}]$ \\
\hline 0,07 & 400 & $>100 \rightarrow$ EMP \\
\hline 0,1 & 700 & $>100 \rightarrow$ EMP \\
\hline 0,2 & 400 & 70 \\
\hline 0,9 & 300 & 70 \\
\hline 2 & 300 & 60 \\
\hline 5 & 300 & 65 \\
\hline 10 & 300 & 60 \\
\hline 20 & 300 & 70 \\
\hline 50 & 400 & 75 \\
\hline 100 & 1000 & $>100 \rightarrow$ EMP \\
\hline 200 & 1500 & $>100 \rightarrow$ EMP \\
\hline
\end{tabular}

Tab I: Measurement results of the spark gap experiments in the vacuum vessel. The first column shows the vacuum pressure, the second the applied voltage, the third the measured current.

EMPs could be recorded only for pressure values $0.07 \mathrm{hPa}, 0.1 \mathrm{hPa}, 100 \mathrm{hPa}$ and $200 \mathrm{hPa}$. For the pressure values in between, a constant current was flowing, obviously with a stable glow discharge without sparks and without EMP. 


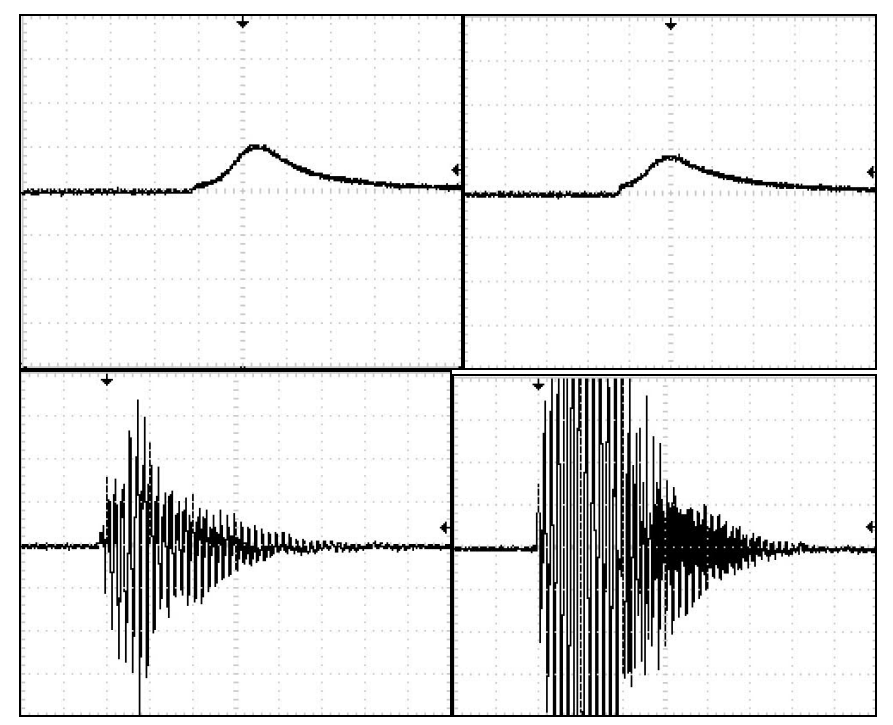

Fig. 9: Measured antenna voltages (y-axes, $200 \mathrm{mV} / \mathrm{div}$ ) versus time (x-axes, $10 \mu \mathrm{s} / \mathrm{div}$ upper two plots, $250 \mathrm{~ns} / \mathrm{div}$ lower two plots). The corresponding gas pressures were $0.07 \mathrm{hPa}$ (upper left), $0.1 \mathrm{hPa}$ (upper right), $100 \mathrm{hPa}$ (lower left), $200 \mathrm{hPa}$ (lower right).

Fig. 9 shows the results of the measured time traces of the EMP, as measured with the antenna for the corresponding 4 pressure stages as mentioned above. For low vacuum pressures (upper two plots), smooth and broad signal curves were obtained, lasting for about $30 \mu \mathrm{s}$ each. This behaviour is interpreted as the ignition of a quient and stable glow discharge which lasts until the power supply limits the electrical current and terminates the discharge. For the high vacuum pressures (lower two plots) strong oscillations are observed that last for about $1250 \mathrm{~ns}$ each. Due to the high amplitude of the received signal (the sensitivity of the antenna arrangement is the same in all cases) such a behaviour might be most appropriate for the detection of sparks and EMP's in such a large system as $\mathrm{W} 7-\mathrm{X}$ is.

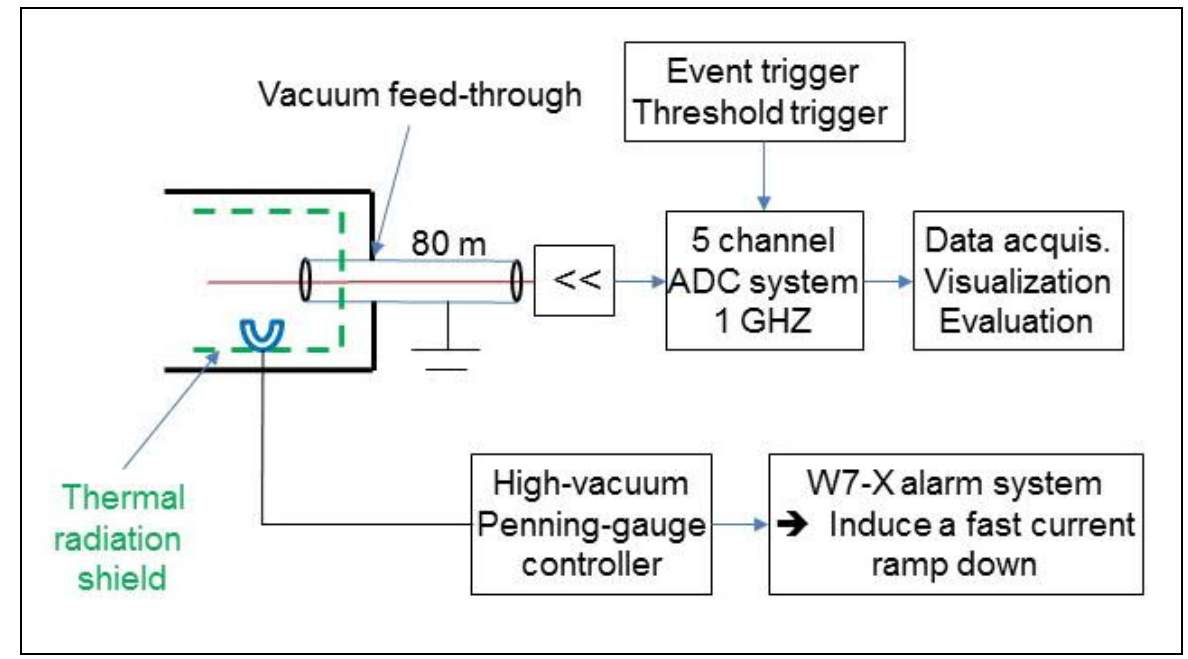

Fig. 10: Sketch of the HF antenna arrangement installed in the W7-X cryostat. Five rod antennas receive the EMP energy (left side) and transfer it via coax-cables to a 5-channel ADC-system. In parallel, Penning gauges are installed to measure the vacuum pressure in the cryostat.

During all the tests performed it turned out that the EMP that occurs during a spark, even at low currents $<100$ $\mu \mathrm{A}$, is a rather characteristic and well detectable phenomenon. Therefore, we focused for W7-X on the development of an antenna system that is simple and robust enough to allow for a minimally invasive installation into the existing cryostat. Essential technical boundary conditions, like maintenance-free operation, vacuumtightness, minimum heat load to the cryostat, no interference with all other electronic systems could be fulfilled after some efforts.

Rod antennas with $25 \mathrm{~cm}$ length are chosen as detectors. To provide, at least rudimentarily, the possibility to localize possible sparks, five antennas are installed in equal distances around the W7-X torus. To match the high impedance of the antennas to the $50 \mathrm{Ohm}$ cables, broadband amplifiers GHV530 are used, providing an 
amplification of $30 \mathrm{~dB}$. The fast signals (bandwidth $1 \mathrm{GHz}$ ) are recorded by a fast 5-channel analog-to-digital converter that can be triggered by hand, or by setting to a voltage threshold to allow for event triggering. Preceding spark experiments in test tanks and the W7-X environment revealed dominating rf-emission bands during sparks around frequencies of about $30 \mathrm{MHz}, 80 \mathrm{MHz}, 100 \mathrm{MHz}$ and $150 \mathrm{MHz}$. Careful impedance matching of the antennas, connectors and cables turned out as essential to optimize the receiving characteristics of the entire system. Fig. 10 shows a sketch of that installation, which is now permanently active during the W7$\mathrm{X}$ coil operation.

In order to control the proper functioning of the antenna system, tests are performed on a regular basis using a network analyzer system Keysight ENA E5080A with a bandwidth of up to $9 \mathrm{GHz}$ and up to 4 ports. For these tests, a low input voltage at variable frequency is applied to one antenna as a sender; the other antennas receive that signal after attenuation within the W7-X cryostat. The attenuation curves are recorded to make sure that all antennas receive a signal as a function of the sender frequency.

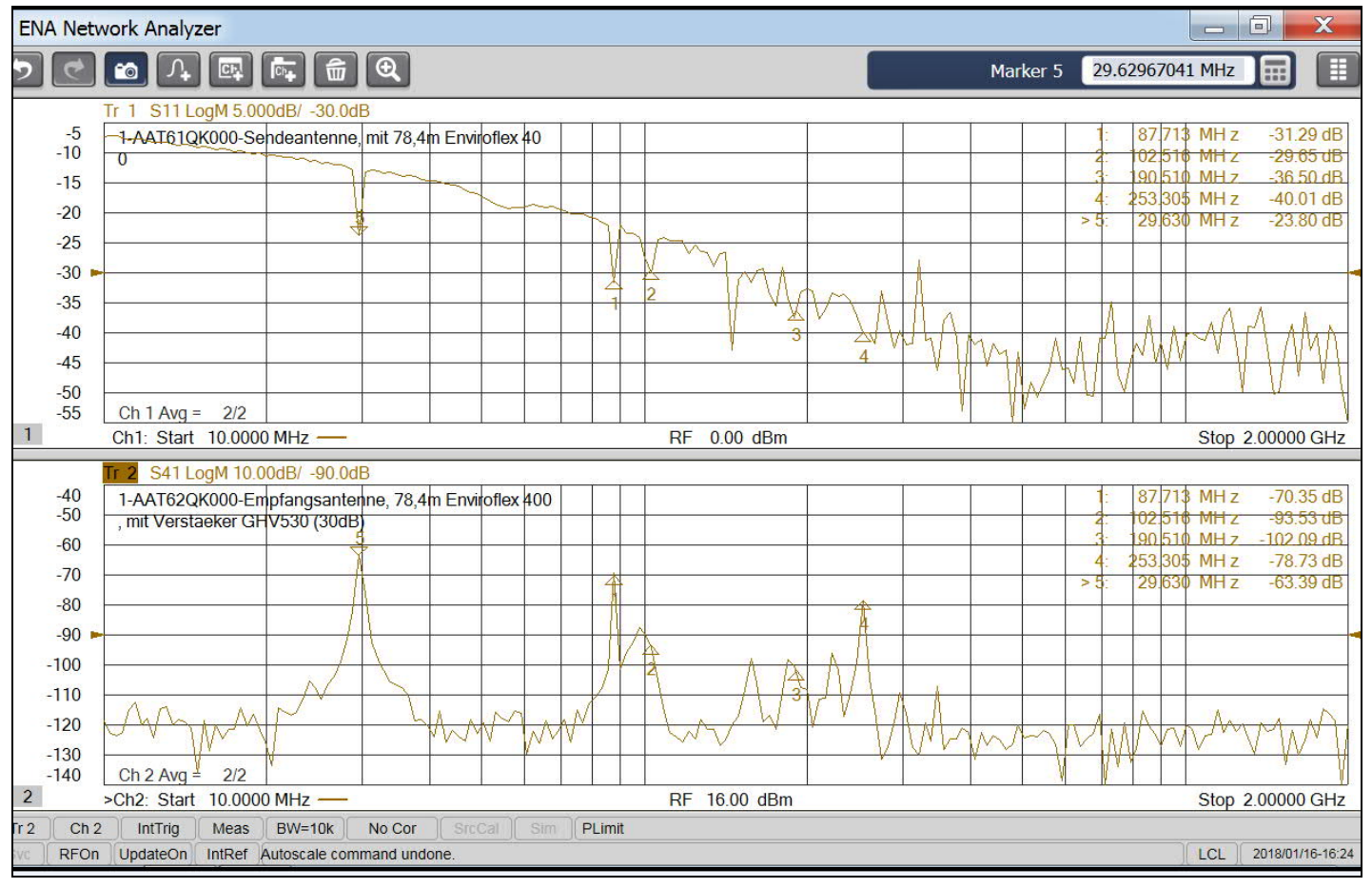

Fig. 11: Result curves from the network analyzer. Upper plot: Emitted power versus frequency for the sending antenna in module 1 in W7-X. Lower plot: Received power versus frequency for the receiving antenna in module 2 in W7-X. Power levels are given in dB.

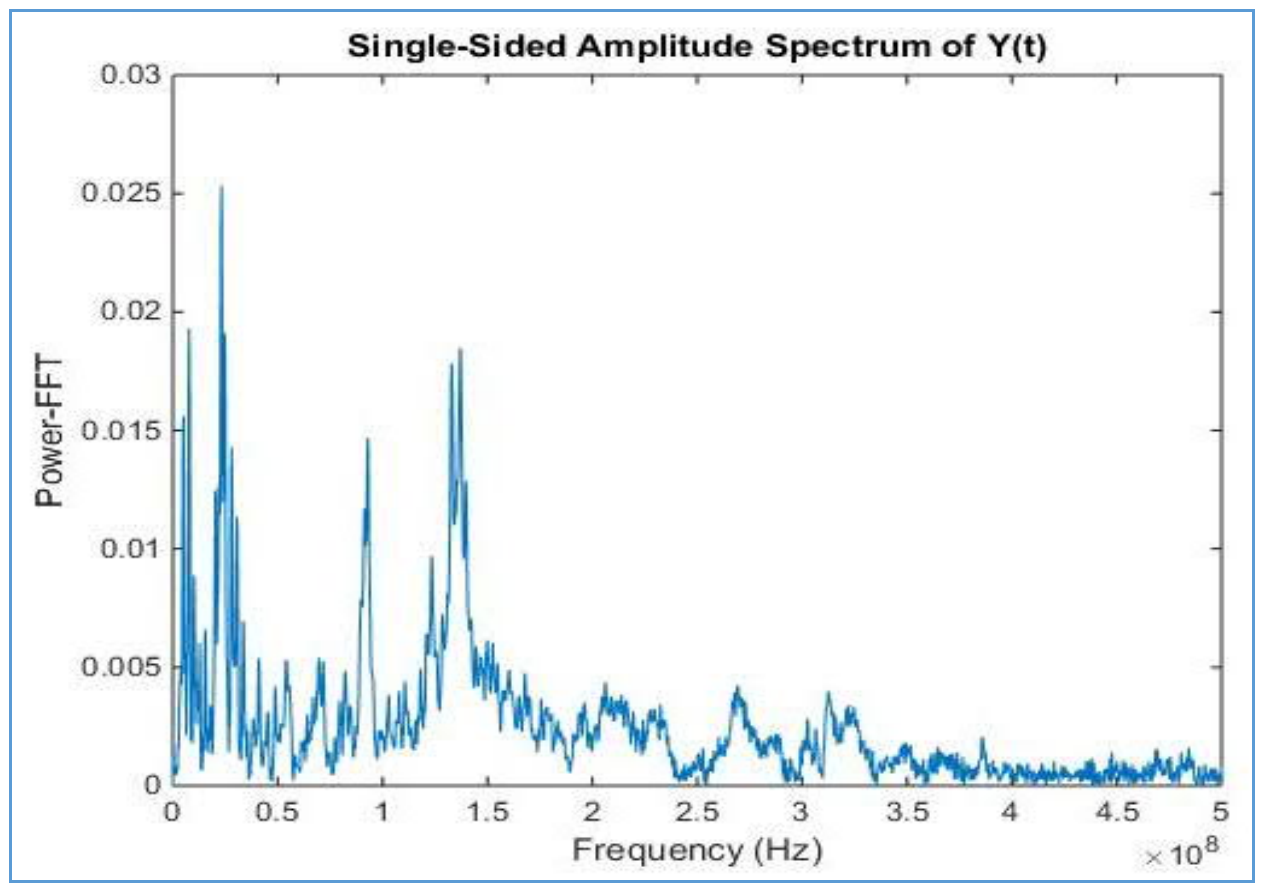


Fig. 12: Power spectrum of the EMP signal. This was measured in the test tank in the lab during an attempt to mimic sparks in W7-X. The power (y-axis) is in arbitrary units, calculated by power-FFT. The $\mathrm{x}$-axis shows the frequency.

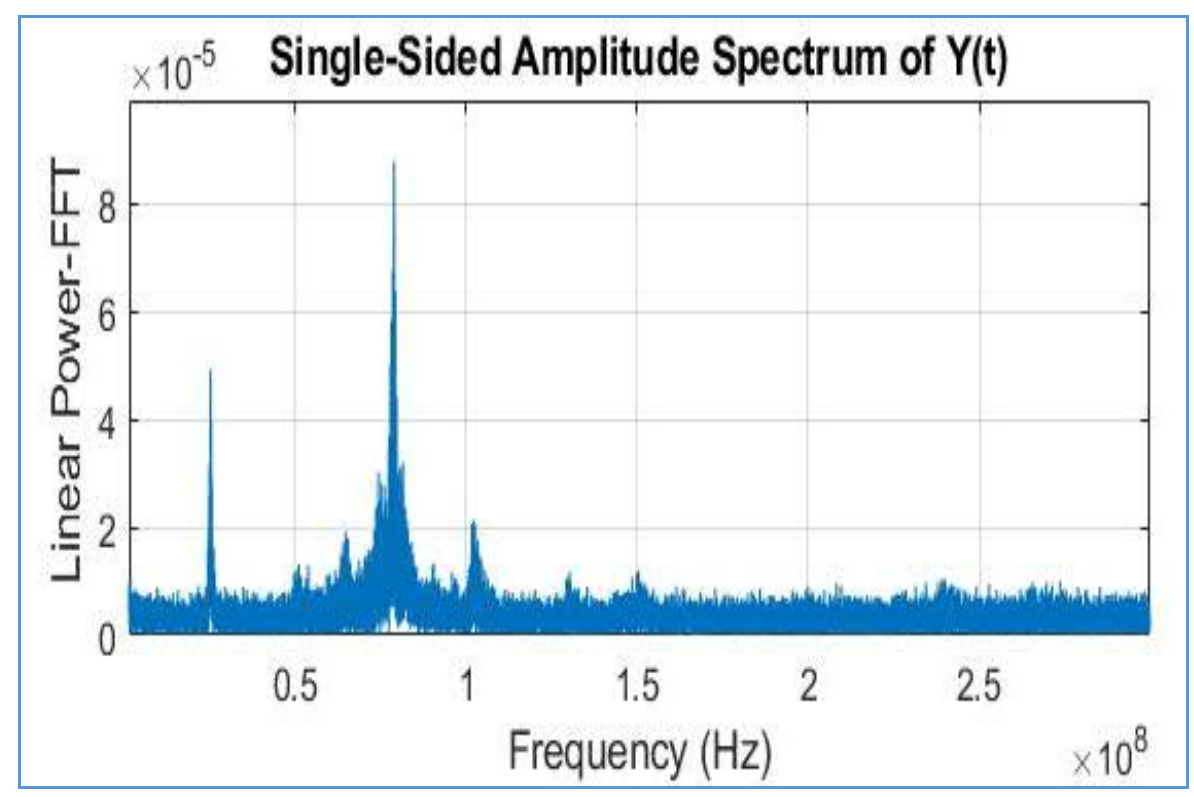

Fig. 13: Power spectrum of the EMP signal, measured with a spark gap connected to a W7-X qd-wire. The power (y-axis) is in arbitrary units, calculated by power-FFT. The X-axis shows the frequency. This shape of spectrum is supposed to come next to the EMP emission during real coil tests in $\mathrm{W} 7-\mathrm{X}$.

Fig. 11 shows the window of the network analyzer output software, in the upper part the sender spectrum of the input voltage, as applied to one antenna in the W7-X module no. 1. The lower part shows the received intensity of one antenna, being situated in module no. 2. One can see drops in the sender power at about $30 \mathrm{MHz}, 90$ $\mathrm{MHz}, 100 \mathrm{MHz}, 150 \mathrm{MHz}$, indicating that at these frequencies power is emitted. These frequencies correspond roughly to maxima in the received power at roughly the same frequencies. We deduce from that image, that the receiving characteristics of the entire system (antennas, cryostat, amplifier, and cablings) are optimum at the frequencies mentioned. Fig. 12 shows the EMP spectrum of a spark, as produced in the test tank in the lab (see below) with comparable spark geometry and energy, as expected during a failed test in W7-X (energy $\approx 1 \mu \mathrm{J}$, length of the spark $\approx 0.5 \mathrm{~mm}$, length of the antenna $0.2 \mathrm{~m}$, test voltage between $1 \mathrm{kV}-2 \mathrm{kV}$ ). Hence, it was tried to mimic sparks in W7-X as good as possible. This spark spectrum shows maxima of the received EMP power at about $30 \mathrm{MHz}, 90 \mathrm{MHz}$, and 130-140 MHz. This is close to the transmission maxima, as shown in fig. 11. Fig. 13 shows the EMP spectrum of a spark, as measured in the W7-X environment with a spark gap connected to one of the qd-wires and a high-voltage applied to the corresponding series of coils. The antenna length was 0.2 $\mathrm{m}$, too. This spark spectrum shows maxima of the received EMP power at about $30 \mathrm{MHz}, 80 \mathrm{MHz}, 105 \mathrm{MHz}$ and $150 \mathrm{MHz}$. Therefore, we conclude that we have frequency windows at about $30 \mathrm{MHz}, 80-100 \mathrm{MHz}$ and $140-$ $150 \mathrm{MHz}$, where the sending/receiving/transmission rate is maximum and therefore optimum for the detection of a spark EMP signal in W7-X. Fortunately, no spark was recorded after the establishment of the methods described here in W7-X; therefore, we can present no example with a real discharge. 


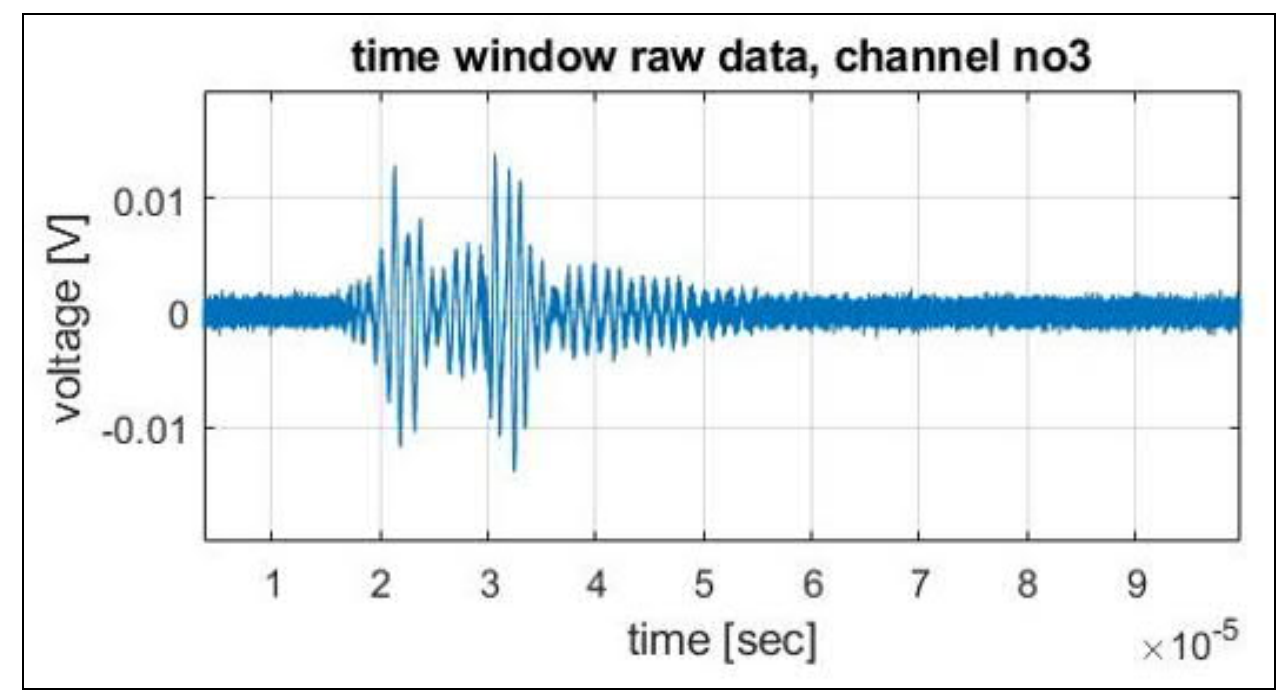

Fig.14: Time traces of an EMP recorded at the end of a $500 \mathrm{~V}$ insulation test, measured in module 3.

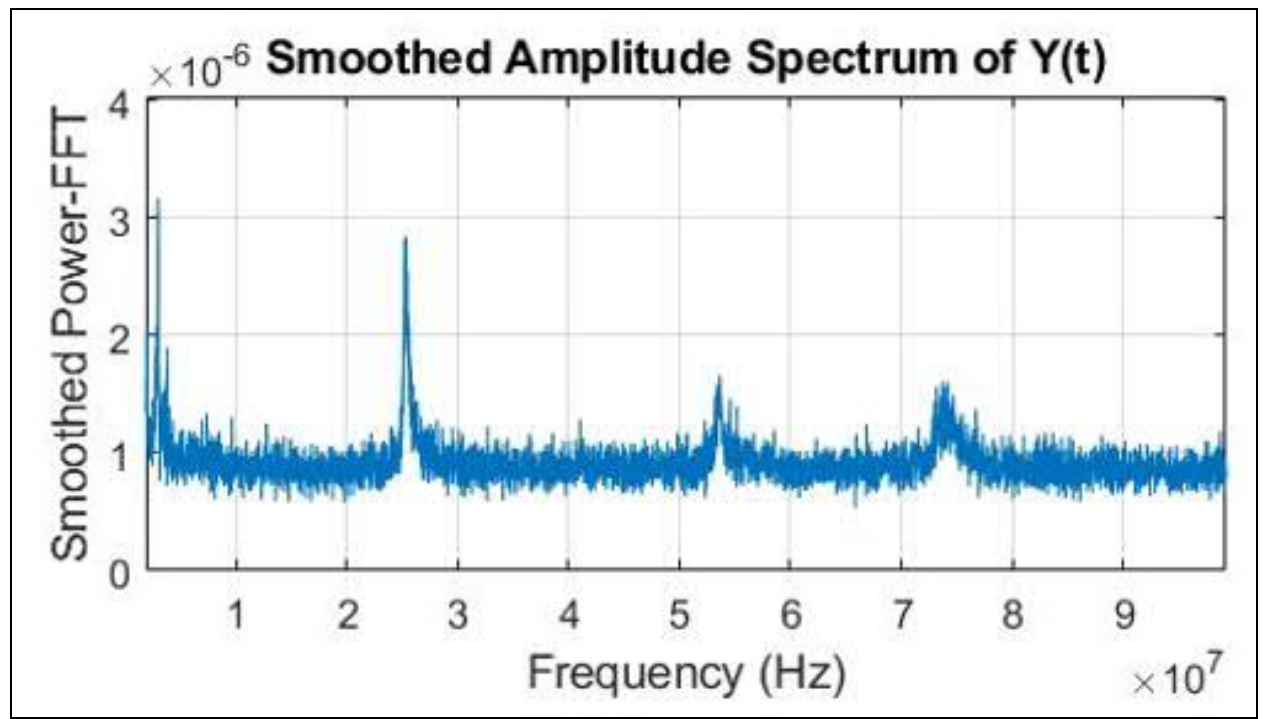

Fig. 15: Power-FFT spectrum of the EMP signal shown in fig. 14. The x-axis is the frequency, the $y$-axis the FFT power in arbitrary units.

A proof for the good sensitivity of the antenna system is given now. The fig. 14 shows the time trace of channel 3 in module 3, recorded during a $500 \mathrm{~V}$ insulation dc-test of the coil system. At the end of the test, the voltage is ramped slowly to zero. Then the dc-source is de-coupled and the series of coils is connected to machine ground. Residual charges in the stray capacity of the coils are then dumped to ground. That discharge can also produce a weak EMP, which can be recorded, however at a much lower signal level than a discharge of the full testing current with full dc voltage applied. Nevertheless, a clear signal can be seen. The fig. 15 shows the power spectrum of that signal, with dominant contributions at frequencies of 26 about MHz, $54 \mathrm{MHz}$ and $75 \mathrm{MHz}$. The match to the frequencies determined above is not perfect, however good enough to allow for the detection of even very weak signals. Therefore, we conclude that EMP's produced during a real dc-test will be registered and recorded.

The insulation vacuum in the cryostat is permanently surveyed by vacuum gauges. However, the residual gas conductance between the coil compartment and the gauges is very poor. To improve that conductance, five Pfeiffer Penning-gauges IKR070 are installed at the same five locations as the rod antennas. Holes in the radiation shield provide a direct gas connection to the gauges, such that any pressure change in vicinity to the coils is measurable with a minimum time delay. The reading of these gauges is directly provided to the central W7-X safety system, such that during every unexpected pressure increase a fast current discharge can be initiated, ideally before a quench occurs. 


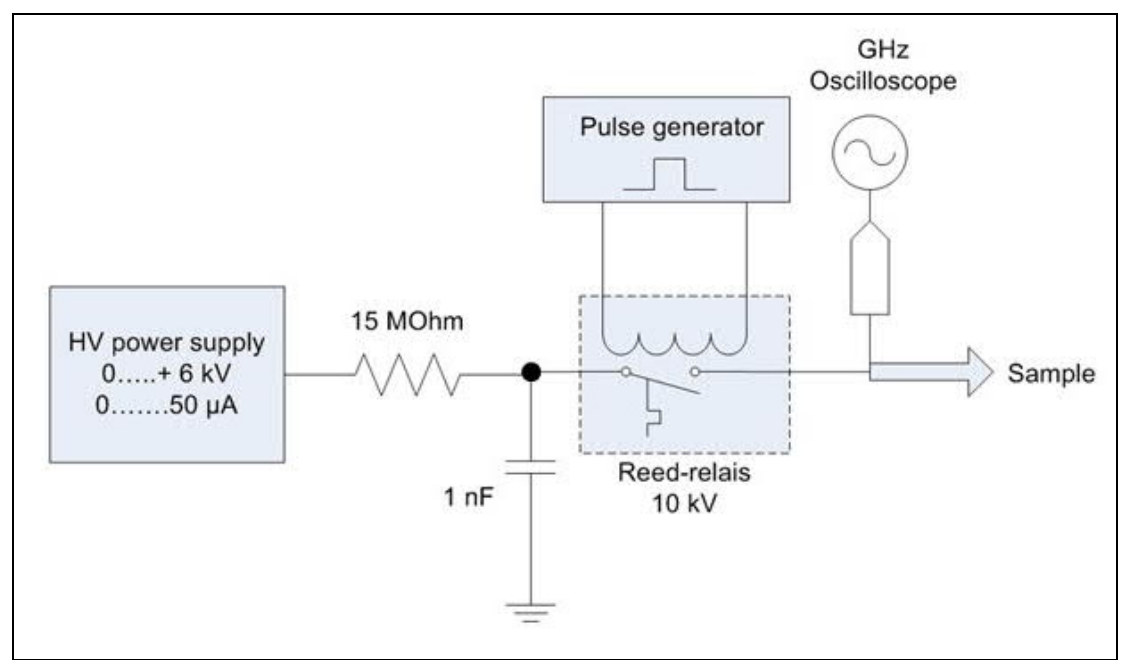

Fig. 16: Sketch of the high-voltage feeding switch used to apply HV-pulses to the coils. The HV is supplied (left side) and applied along an insulation resistor (typically $15 \mathrm{MOhm}$ ) to the sample (right hand side).

A technique for the localization of possible weak insulation points employs the application of fast HV pulses to the coil system. The system response is recorded with high time resolution by an oscilloscope. Fig. 16 shows the principle of the HV pulse sender. The bandwidth of the oscilloscope must be in the GHz range. The system response can be evaluated as described now.

The solution of the telegrapher's equation [8] provides, at least in principle, the means to localize potential highvoltage insulation defects along a cable. The idea behind is to apply a high-voltage pulse with steep leading edge to the cable. If a discharge from the conductor to ground occurs at a certain location, the response can be recorded after a certain delay time. The measurement of that delay time provides information on the localization of the insulation defect. Each cable or coil can be represented as a series of serial / parallel resistors $R(x) / G(x)$ and inductances $L(x)$ with stray capacities $C(x)$, all quantities being a function of the cable length $x$ and time $t$. According to Kirchhoff's law the telegrapher's equations look like: $d U(x, t) / d x=-L \cdot d I(x, t) / d t-R \cdot I(x, t)$ and $d I(x, t) / d x=-C \cdot d U(x, t) / d t-G \cdot U(x, t)$ for the voltages $U(x, t)$ and currents $I(x, t)$.

A propagating wave being described like $U_{0} / I_{0} \cdot \exp \{\mathrm{i}(\omega t \pm k x)\}$ solves the equations for the wave frequency $\omega$ and group velocity $\mathrm{V}_{\text {group }} \approx c / \operatorname{sqrt}\left(\varepsilon_{r} \cdot \mu_{r}\right)$ with amplitudes $U_{0}$ and $I_{0}$ for voltage and current, respectively. For a co-axial shielded cable under vacuum we have the magnetic permeability $\mu_{r}=1$. The dielectric constant is $\varepsilon_{r}=2.8-3.8$ for Capton ${ }^{\circledR}$ with the speed of light $c$. The abbreviation "sqrt" in the formula above stands for the square root. Capton ${ }^{\circledR}$ is used for the quench-detection wires inside the coil cryostat. This provides typical propagation velocities of a voltage front (group velocity of a propagating wave) of $\mathrm{V}_{\text {group }} \approx 0.2 \mathrm{~m} / \mathrm{ns}$.

The localization of insulation defects, for instance along the quench detection wires, was performed successfully. The insulation could be repaired in all discovered weak-insulation cases prior to closing the cryostat. Fig. 17 shows an example of the voltage traces as a function of time, for the comparison between a damaged and one undamaged wire. 


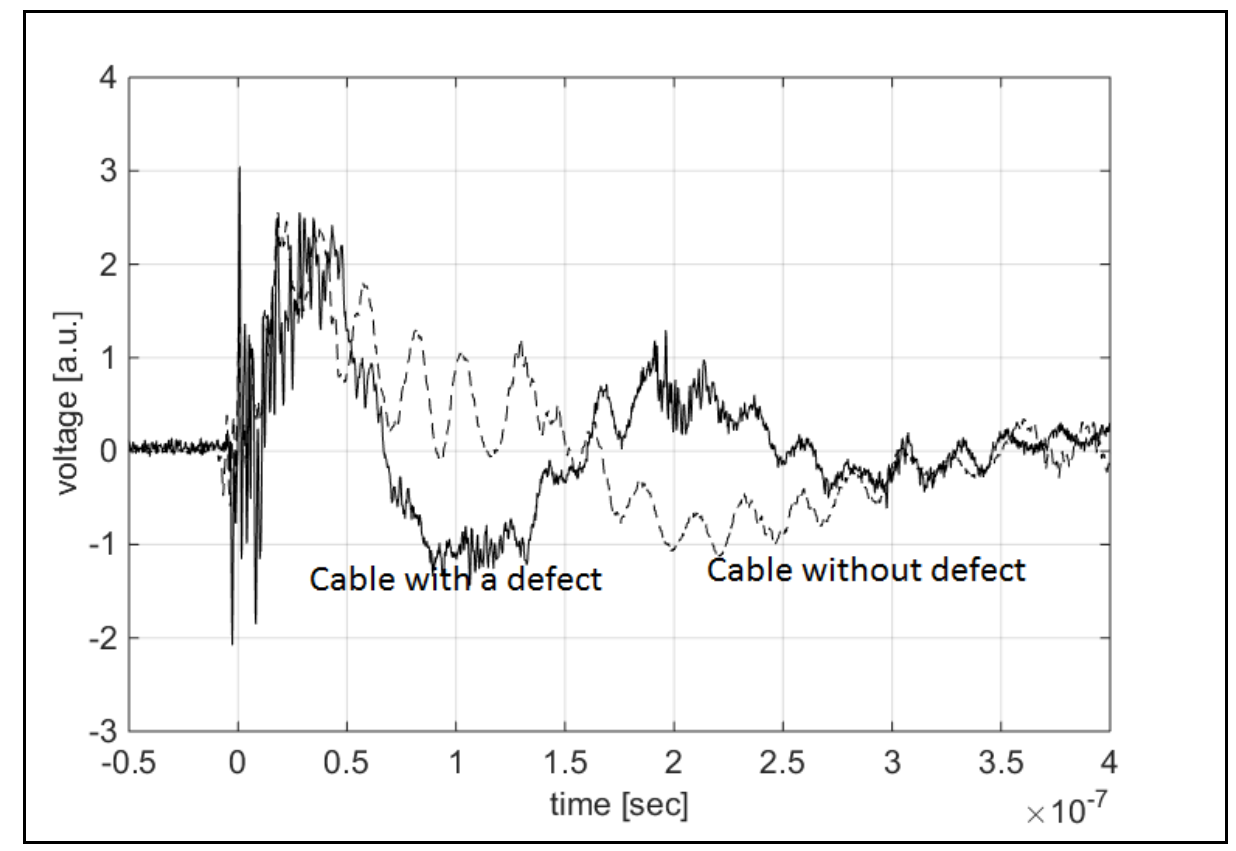

Fig. 17: Example of two high-voltage reflection curves. The solid line shows a cable with an insulation defect, compared to another cable without defect (dashed line). The curves start to differ after about $15 \mathrm{nsec}$, i.e. the defect is about $3 \mathrm{~m}$ away from the cable entrance. The HVpulse is applied at $\mathrm{t}=0 \mathrm{~s}$.

However, the formation of a spark at the location of the defect is inevitable, possibly aggravating the existing defect even more. In addition, only individual wires can be examined with unequivocal results. In case of the W7-X coils, a cable network is realized with numerous branches and interconnections. This imposes severe restrictions to this method if the defect is situated, for instance, behind a cable intersection.

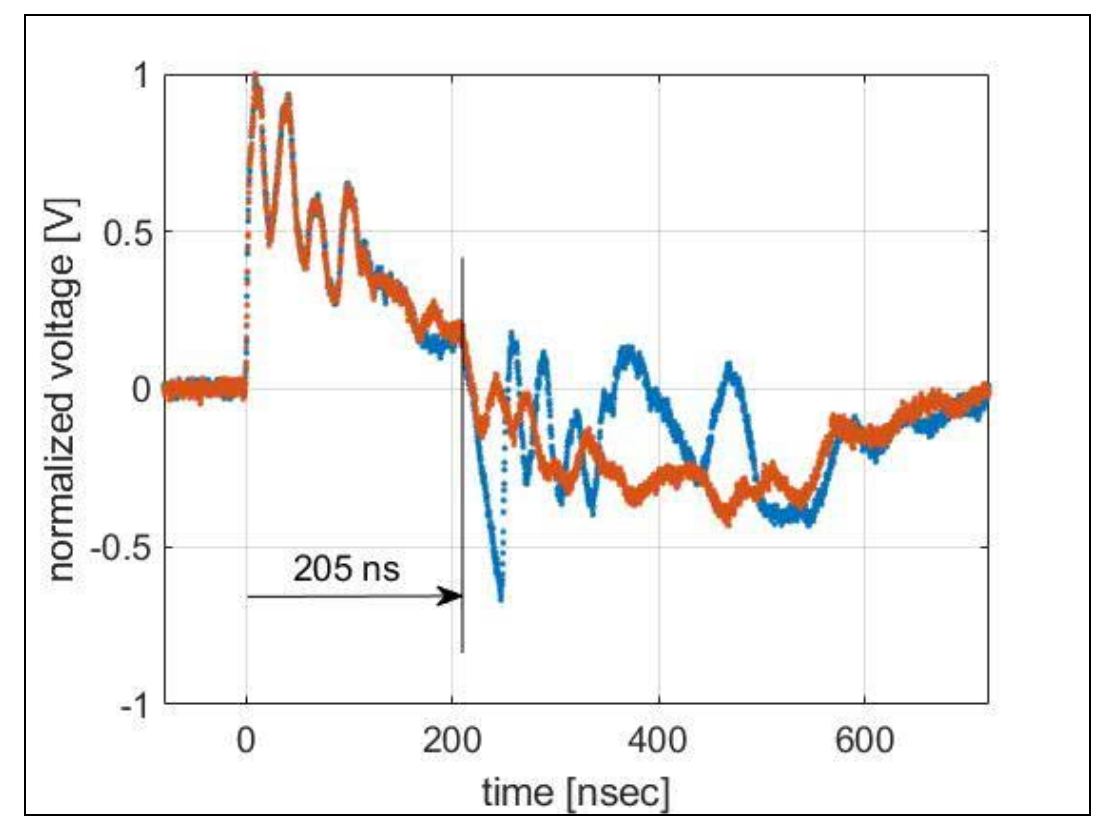

Fig. 18: Time traces of the high voltage response after application of a hv pulse to two series of coils. The voltages are normalized to each other, in both cases $2500 \mathrm{~V}$ were applied. The red curve shows the voltage response of the series of coils that passed the dc-test; the blue curve was measured on the series of coils that failed. Colors are visible only in the online version of the paper.

Fig. 18 shows time traces of the high voltage response measured on series of coils. The voltages are normalized to the same level for plotting; the input voltage was $2500 \mathrm{~V}$. One series of coils shows a sort of break-through after a time delay of about $205 \mathrm{~ns}$, indicating a weak insulation point about $20 \mathrm{~m}$ away from the voltage feed point (blue curve). This coil group failed a dc high-voltage test briefly before the pulse test. The break-through becomes obvious only when the time curve is compared to another coil group that passed the same dc-test before 
(red curve). The two voltage curves are almost identical to each other until $\approx 205 \mathrm{~ns}$, and then they deviate from each other. However, as during superconductor operation and fast current ramp-down only max. $950 \mathrm{~V}$ can be reached (see above); this test was never repeated at $2.5 \mathrm{kV}$. At $2 \mathrm{kV}$ dc testing voltage, the coil group under consideration passed the test. The same coil group was investigated with the method of a slowly rising dc highvoltage, as described below. In order to avoid further damage on this coil group by testing with such elevated voltages, the further tests were all performed at lower voltages $<2.5 \mathrm{kV}$, where this coil group succeeded, fortunately.

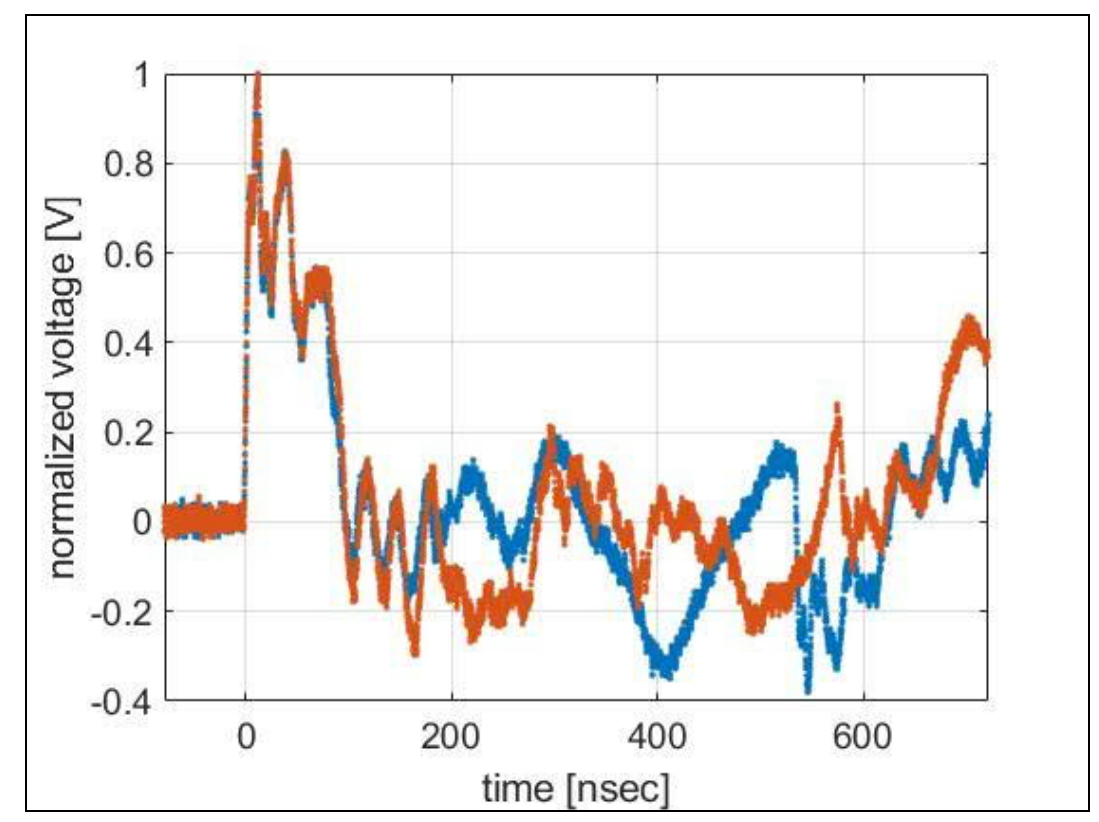

Fig. 19: Time traces of the high voltage response after application of a hv pulse to two adjacent qd-wires on one series of coils. The preceding dc high-voltage test was passed. Until about $200 \mathrm{~ns}$, the two curves are almost identical, but then they deviate from each other as a result of different cable branches.

Fig. 19 shows high-voltage time traces for one series of coils, where the high-voltage pulse was applied to two adjacent qd-wires. The applied voltage was in both cases $2500 \mathrm{~V}$. During preceding dc-tests that series of coils passed successfully, hence no weak insulation points are expected. However, the direct comparison of the two traces show that the two signals are almost identical up to about $200 \mathrm{~ns}$, then they start to deviate from each other considerably. In this particular case, the deviation is a result of different branches along the individual qd-wires, rather than an indication for a weak insulation point. This shows, in comparison to the result in fig. 18, that not only a current leak will lead to different voltage response curves, but also different cable branches and interconnections along the cables. This makes the results of this procedure, if performed alone, ambiguous.

For another approach, the high-voltage is fed to one cable input, but the voltage is slowly increased within about one minute to a maximum value. The voltage rise has to be slow enough such that the charging current into the stray capacities does not exceed the upper current test limit. A number of sensor cables (all of equal length) connect the same number of measurement points along the superconductor to the input channels of a fast oscilloscope (in our cases max. 4 channels). In case of a spark, the high-voltage will break down locally at the weak point, and a propagating wave will be induced, moving away from the location of the spark. This falling voltage front/wave will propagate along the coils/cables until the oscilloscope records it after a characteristic time delay. By measuring the relative time delay between the channels it is possible to infer roughly where the defect spot is localized.

The precision of this method is rather limited because the coil series act as an electronic low-pass filter, and cable branches inside the coils introduce ambiguities into the recorded signals. Only low frequency contributions travel far enough to be recorded. In practice, the precision in localizing cable defects turned out as being in the range of a few meters. 


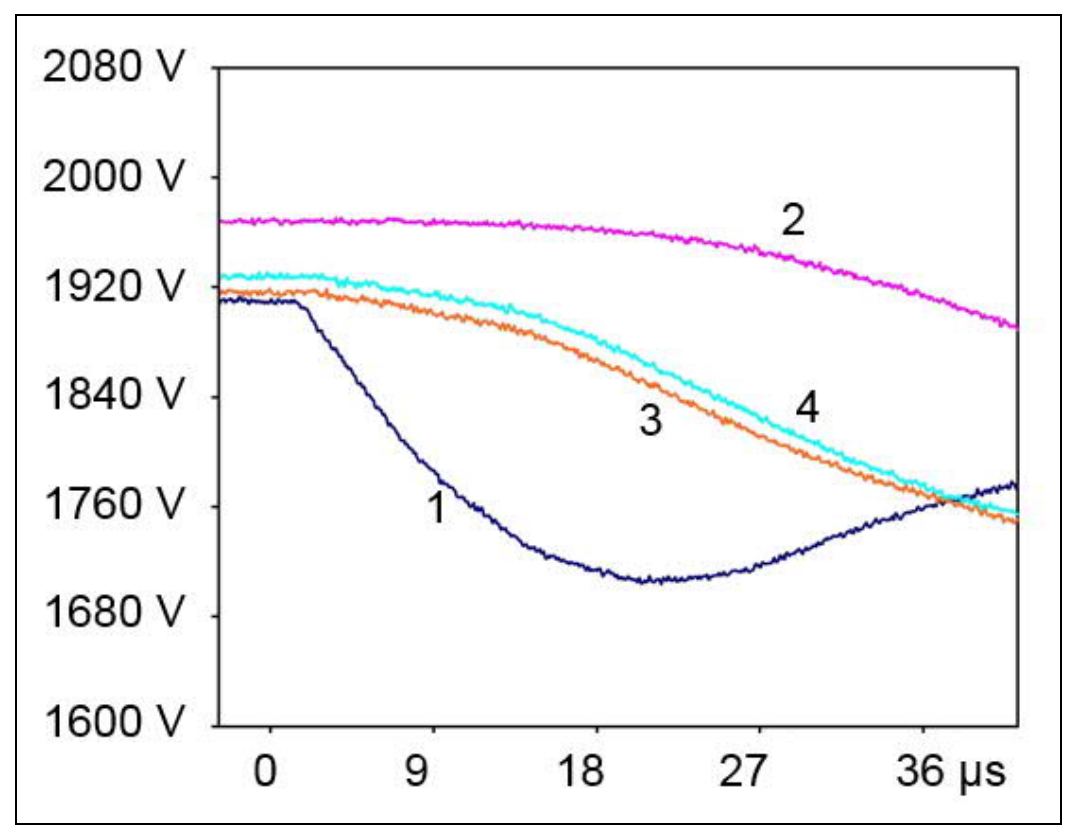

Fig. 20: Result of the run-time method were a slowly rising dc high-voltage is applied to the series of coils in W7-X, until a discharge occurs. The $\mathrm{x}$-axis shows the time, the y-axis the voltage as detected on four channels of a fast oscilloscope. In this case, the 4 input channels labelled $1-4$ were connected to qd-wires as shown if fig. 6 above

Fig. 20 shows the result of the run-time method for the case, that the four oscilloscope channels are connected to the coil group as indicated in fig. 6. Obviously, channel 1 reacts first with a decreasing voltage after the discharge, followed by channel 3 and 4, and channel 2 is the last one in the sequence. This time behavior allows inferring directly to the location of the weak insulation point. By changing the four connection points iteratively and step-by-step, it is in principle possible to encircle the current leak. In this case, the results point to a defect close to the coil in module 1 . As the series of coils act as an electronic low-pass filter, the higher frequency components of the voltage step are filtered away, the further the propagation front travels. This smears out the measured voltage curves and imposes restrictions to the precision of that type of measurement, as can be seen in fig. 20. The later the voltage front arrives, the longer is the voltage decay time and the more diffuse is the point of onset of the voltage decay.

In a test tank in the laboratory (see below), it is also tried to localize insulation defects for the case, that the applied voltage ignites no spark. This should reduce the testing stress to the insulation. The idea is that weak insulation points, for instance after insulator delamination or minor damages, might exhibit a local step in the insulator dielectric constant. This gives rise to deviations in the system response, when comparing them to undamaged cables [9]. We can make use of the fact, that W7-X has seven coil series that can be compared to each other. In addition, the testing voltage can be kept low in order to minimize the deleterious impact of the test itself, and that minimizes the risk of provoking new insulation damages. The data interpretation turned out as not so straight forward because of the missing spark.

A RCL measurement bridge (for instance a Hioki 3522-50) with an operating voltage of only $1.5 \mathrm{~V}$ is used to measure the complex impedance of the coils for frequencies between $10 \mathrm{~Hz}$ and $100 \mathrm{kHz}$. It turned out, that the measurement results are highly sensitive to defects in the insulation, but also for winding errors or short-circuits. Fig. 21 shows the result of typical measurement curves. Those curves are compared to numerical simulations of the coil behavior, using appropriate equivalent circuits of the coils, performed either with the ANSYS or the SIMPLORER software [10]. Such an equivalent circuit is shown in fig. 22, combining the most prominent electronic properties that characterize the superconductor. 


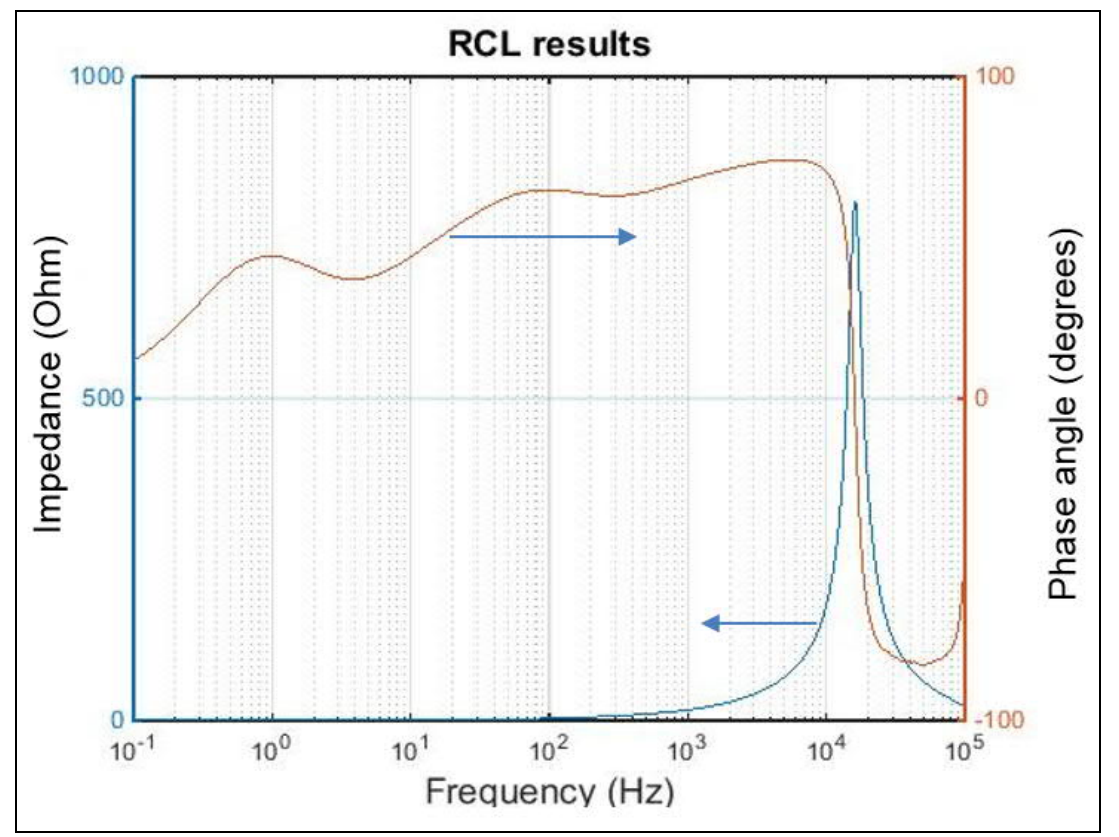

Fig. 21: Result of the impedance measurement of a single coil, showing the absolute value of the impedance (left y-axis), the U-I phase angle (right y-axis) versus the test frequency (x-axis) for an intact coil. Colors are visible only in the online version of the paper. The arrows indicate the assignment of the curves to the axes.

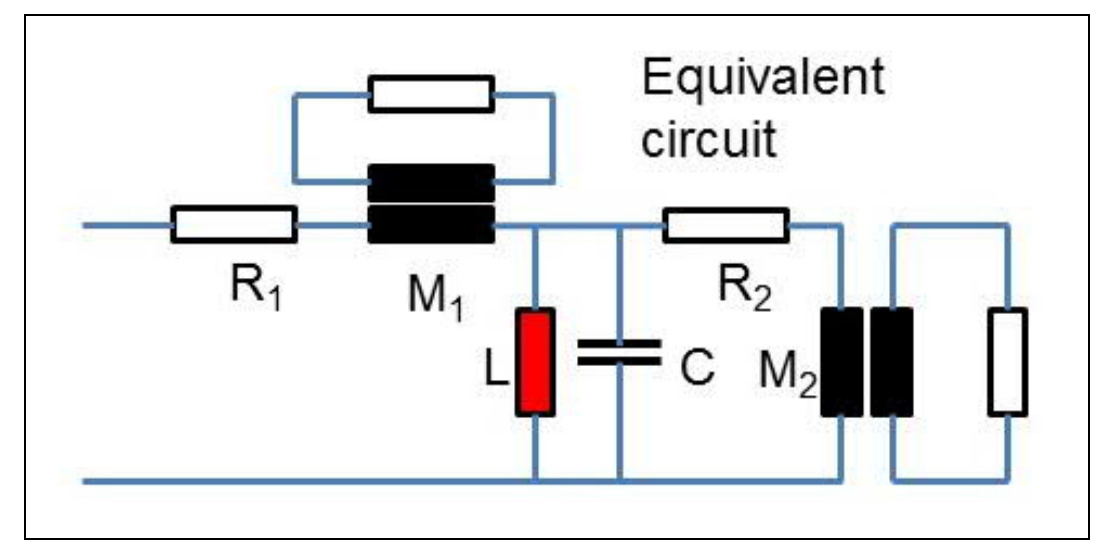

Fig. 22: One of the equivalent circuits, as used for numerical simulation of the coil behavior. The superconductor inductance $\mathrm{L}$ is in parallel to the stray capacity $\mathrm{C}$ originating from the coil casing and the vessel wall, some resistors $\mathrm{R}$ stem from the normal conducting parts of the current connectors, and some ohmically charged transformers M1 and M2. Those transformers exist, because the coil windings are enclosed by the coil casing, the radiation shield, and the other coils. 


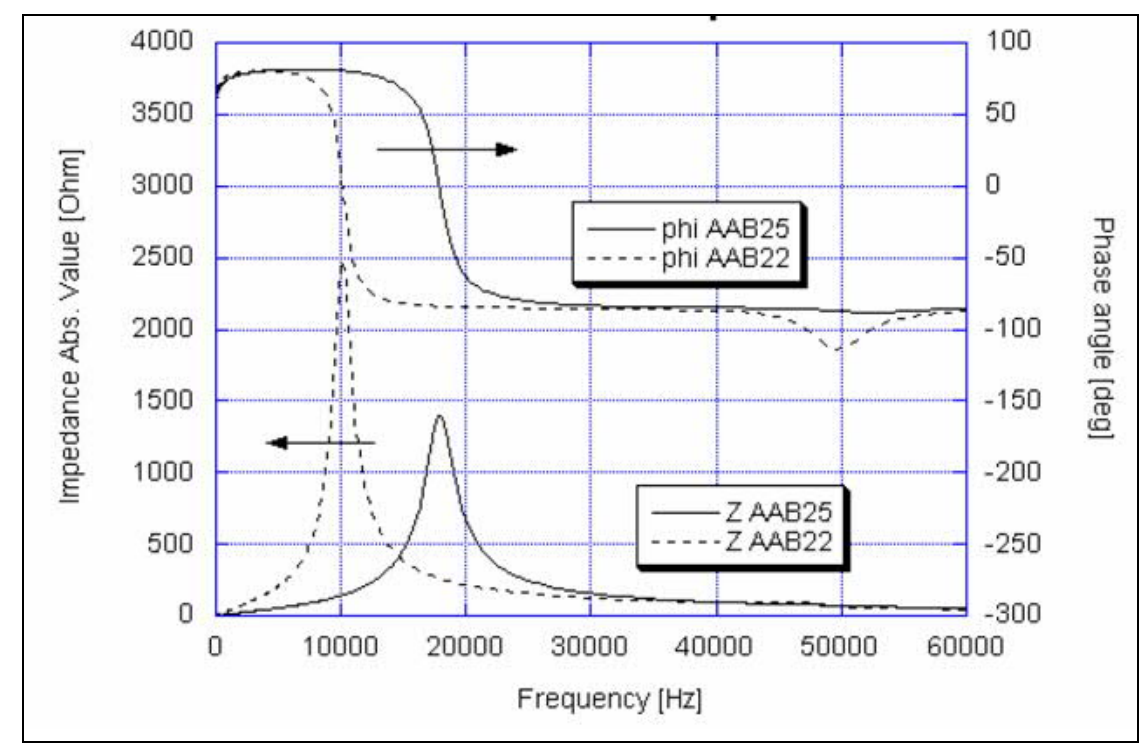

Fig. 23: Measured impedances of two coils (labelled to as AAB25 and AAB22) versus measurement frequency (x-axis). The left y-axis shows the absolute value of the impedance, the right $y$-axis the U-I phase angle. The solid lines show the measurement for the artificially damaged coils, the dashed lines the result for the intact coils.

Fig. 23 shows the measured impedance curves at low voltage for the coils labelled AAB25 and AAB22. For both coils, an artificial current leak versus ground is introduced by connecting one qd-wire to ground. Similar investigations were performed in order to test methods for the detection of short-circuits within the coils, i.e. in between the layers L1 - L12. The impedance curves, both for the absolute value of the impedance (left y-axis) as well as the U-I phase angle (right y-axis) show a considerable difference when comparing the intact coil (dashed curves) to the coil with an artificial damage (solid lines). The measured results could be re-produced well by simulating numerical calculations.

During the routine high-voltage tests, partial discharge (PD) measurements [11] are performed. The "Forschungs- und Transferzentrum Leipzig e.V" conducts that activity, because there the required hardware and skilled personal staff are available. Albeit we use for testing (also under Paschen-minimum pressure conditions) always a dc-source, the phase-resolved PD technique, synchronized to $50 \mathrm{~Hz}$, is employed in order to filter out perturbing sources being synchronized to the $50 \mathrm{~Hz}$ net supply. All registered PD events above a given threshold value are recorded either in time domain, or as a time-correlation histogram to detect correlated PD events. The time-domain reflectometry with single input channels is employed, as well as time-difference measurements with two or three input channels.

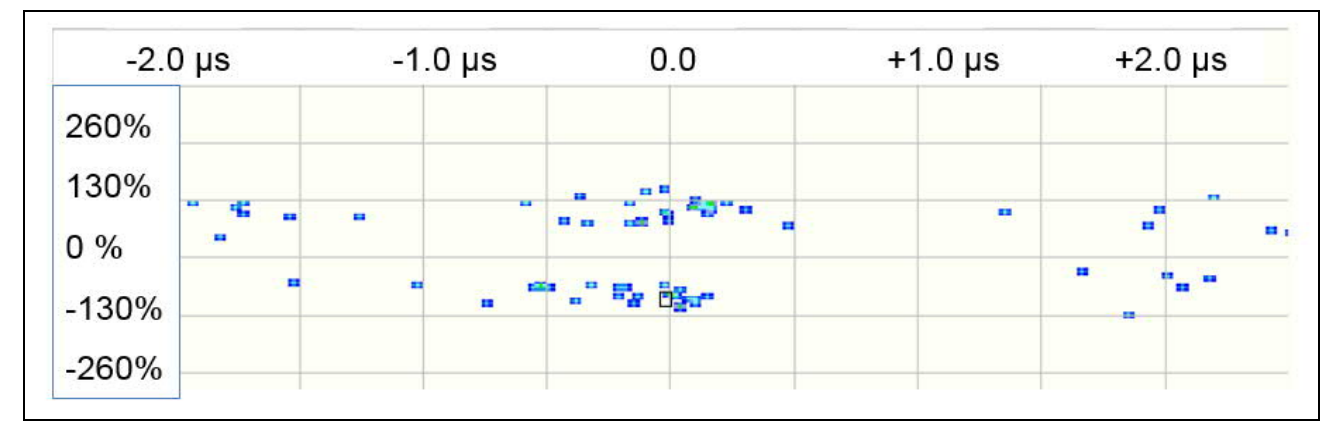

Fig. 24: Time Correlation diagram for a series of planar coils in W7-X, measured with the Partial Discharge equipment. The $\mathrm{X}$-axis shows the time delay in $\mu \mathrm{s}$, the $\mathrm{y}$-axis the measured PD charge in units of $100 \%$ above a pre-selected value.

Fig. 24 shows, just as one typical example, the Time Correlation diagram measured on a series of planar coils during the Paschen tests. The time correlation is taken by measuring with two input channels on the left and right tail-end of the series of coils. A correlation time of $0 \mu \mathrm{s}$ means, that either an event above a certain charge threshold (in this case $80 \mathrm{pC}$ ) occurred in the middle of the series of coils, or an uncorrelated perturbing signal reached both input channels simultaneously. Positive correlation times (x-axis) mean, that the occuring signal was first detected in the triggering channel, negative correlation times the opposite. The y-axis gives the integrated charge of the PD event with respect to $100 \%$ of a pre-selected value ( $40 \mathrm{pC}$ in this case). The background pressure was $0.2 \mathrm{hPa}$ in the cryostat, a dc voltage of $1600 \mathrm{~V}$ was applied. One can see that many events occur close to a correlation time close to $0 \mu \mathrm{s}$, indicating external electronic perturbations. In this case the 
threshold was set close to the overall perturbation level, to maximize the sensitivity against PD events in the coil insulation. All registered events are roughly of the same hight, also indicating that only perturbations are recorded, that penetrated accidentally the threshold. No clear indication is given for an enhanced PD activity within the coil insulation for that particular measurement.

So far, these measurements indicate a broad spectrum of PD activity in the system, however due to the harsh environment and the complexity of the entire coil arrangement, no successful localization of single events could be accomplished. The integral PD level, recorded during all tests so far, is considered as uncritical concerning the quality of the insulation. Nevertheless, long-time measurement series are initiated to observe the PD level in the future and compare it to measurements in the past.

So far we are not able to detect clearly correlated PD events at given time differences with enhanced probability, compared to the statistical level of uncorrelated, purely accidental PD events. On the other hand, these signals as well as the reflection curves obtained by the HV pulse reflection methods seem to contain more information than only noise. In the last chapter IV, we describe numerical attempts to draw more information out of these measurements.

A further electronic test for the rough localization of sparks is presently under consideration. The idea is to connect a high-voltage source to the two connection-ends of a coil series. Then the level of the high-voltage is gradually increased within about 1 minute, until a possible spark ignites at the insulation defect. An external network of resistors provides a bridge circuit, which is connected to the high-voltage supply and the two connection-ends of the series of coils. A fast analog-to-digital converter records the voltage symmetry measured across the bridge during the spark. The resulting symmetry (or asymmetry) of the voltages points to the location of the defect, taking advantage of the fact that the superconductors are connected in a linear series.

\section{Optical methods for spark detection}

If a single testing technique does not provide sufficient information for an insulation repair, a combination of techniques might help us further. Numerous optical methods are tested in a vacuum test tank in the laboratory, because they bear the potential of direct spark observation and localization, provided appropriate access to the cryostat can be realized. Presently, none of these optical techniques is installed on W7-X because of the huge effort. Nonetheless, they are developed and assessed with respect to their usability on W7-X. Thus, the expertise will be available when it is needed.

Different photoelectric sensors (PIN photodiodes, avalanche photodiodes, photomultipliers, CMOS and CCD cameras) are tried in combination with vacuum looking glasses or optical fibers on a test tank. Sparks are ignited in a spark gap, with typical electrical parameters as given during our high-voltage tests $(\mathrm{U}<2 \mathrm{kV}, \mathrm{I}<100 \mu \mathrm{A}$, gap width $<0.5 \mathrm{~mm}$ ). Then the sparks are recorded with the optical sensors.

To come closer to the situation in W7-X, the tests are performed at vacuum pressures around Paschen-minimum conditions, or good vacuum pressure $<10^{-5} \mathrm{hPa}$, and with different working gases. Most promising, so far, is the use of Hamamatsu photomultiplier modules (for instance H10721-210) with an optical fiber connection. They combine high speed and bandwidth with high sensitivity. The use of optical fibers is highly appropriate for the use in our cryogenic environment (low heat conductivity, low outgassing, simple vacuum feed-through, robustness, low volume, no maintenance etc.).

The largest problem during the test is the fact that each spark induces, besides the light, also an EMP that is detected by the sensors. Therefore, the potential of the site-directed sensitivity of optical methods is strongly affected. This holds in particular for very light sensitive cameras, which can detect the weak sparks even in a distance of $5 \mathrm{~m}$. However, the EMP induces electronically a spot on the light sensitive chip, which resembles strongly the visual image of a spark.

Among all detectors tested, only the photomultiplier modules could be shielded perfectly enough to mitigate that effect. This concept includes also the shielding against the permanent magnetic field in vicinity to W7-X, and potential nuclear radiation from the plasma experiment.

Fig. 25 shows a sketch of a testing arrangement within the vacuum test tank, here with the Photomultiplier (PM) modules. This tank has a diameter of about $1 \mathrm{~m}$, hence large enough to contain all required hardware. The pressure inside can be varied, and different testing gases can be applied. Several feed-throughs for cable and optical fibers allow for feeding the installed arrangements, optical windows allow for the testing of different camera types. 


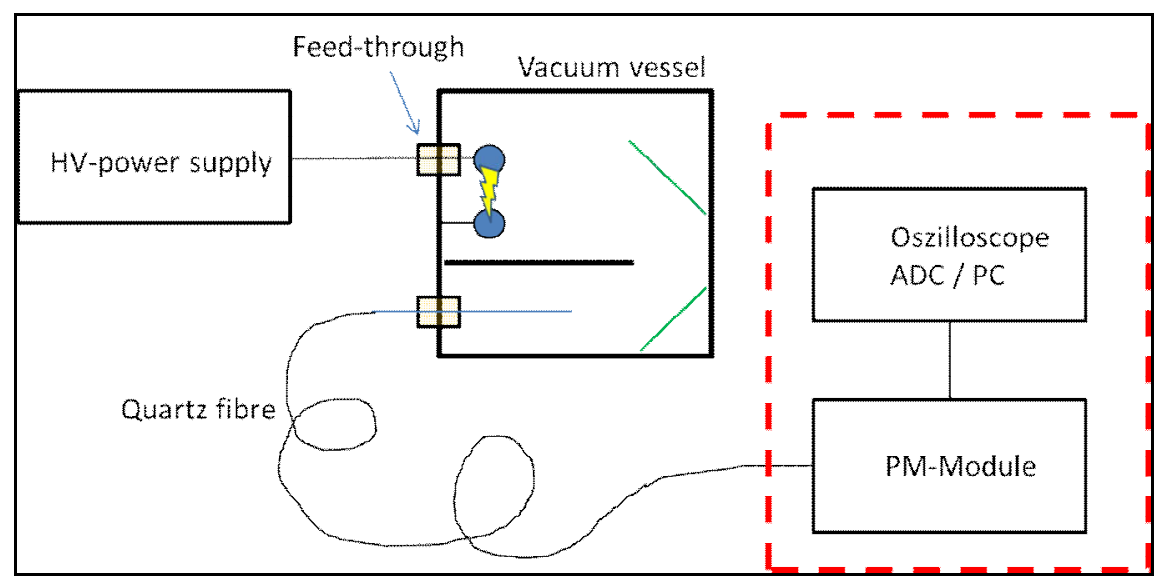

Fig. 25: Sketch of the testing layout for the photomultiplier module inside the small test tank. A light labyrinth inside the vessel simulates the complex geometry inside the W7-X cryostat that might block direct illumination of the fiber.

An alternative optical method is considered for the future, making use of human visual inspection, or the use of the light-sensitive cameras. The challenge is to simulate low-pressure Paschen-minimum conditions, however at open cryostat and 1 bar background pressure in the case that one day sparks occur. In addition, we have the requirement to work at reduced high-voltage, and we want to avoid numerous repetitions of the high-voltage stress on the coils. This all can be realized by the use of transparent plastic bags around all components under suspicion, filled with gases of lower ignition voltage than air. Neon was tested successfully as gas for that purpose, because the HV tests can be performed at considerably reduced test voltage. Visual inspection during the tests should then point directly to the insulation defect. For that step, however, the cryostat has to be opened and holes have to be cut into the radiation shield.

\section{Numerical methods}

When applying a high-voltage pulse to the coil system, the recorded electronic system reply resembles noise, however regular patterns seem to appear within. When comparing the pulse response of different wires, they resemble to each other to some extent, i.e. they seem to be not completely noisy or random. This is supported by cross-correlation calculations performed for the electronic system reply for different quench-detection wires, as shown in fig. 26. The obvious similarities between different voltage time traces are caused by the fact that many cables lie parallel, are connected to closely linked parts of the superconductor, or cross talk between the wires induces similar signals on wires lying close to each other in the cryostat. As fig. 26 shows, the result of the crosscorrelation calculation can exceed a statistical limit, presuming that a random process with a Gaussian noise characteristics is underlying. For the example shown, an upper limit of $\pm 2 \sigma$ absolute value of the standard deviations is chosen arbitrarily.

A more thorough numerical analysis of these responses will therefore be helpful for the interpretation with respect to weak insulation points. The need for such an interpretation becomes obvious when looking at the curves shown in fig. 17: only the direct comparison between them reveals the localization of the defect. If a direct comparison is not possible: are there means to draw more information out of the system when analyzing the voltage time traces? 


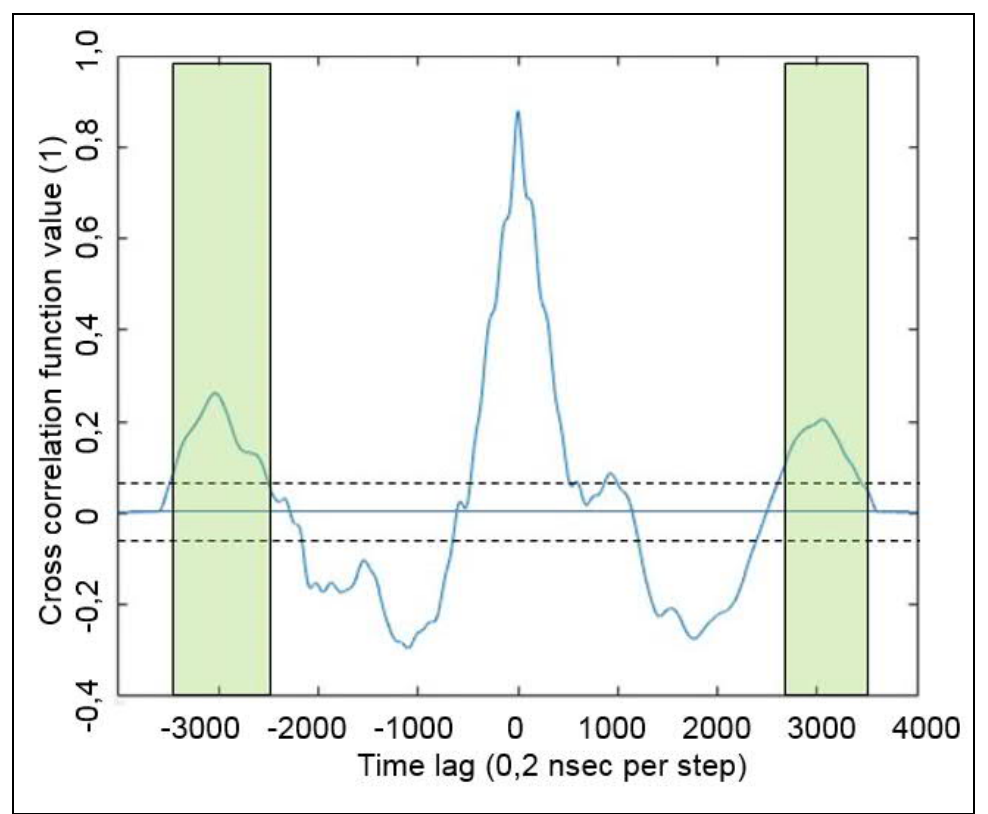

Fig. 26: Normalized cross correlation value (solid line) for the electronic system response of two adjacent quench-detection wires. For a time lag of about \pm 3000 samples $(0.2 \mathrm{~ns}$ per sample along the $\mathrm{x}$-axis, green vertical areas) the correlation exceeds a $\pm 2 \sigma$ threshold (dashed horizontal lines) indicating some systematic correlation between the signals, exceeding that statistical noise level.

Various numerical methods are tested to evaluate and understand the noisy and perturbed measurements from a real existing large coil system in a very rough environment. An additional problem is always to figure out, which phenomena are caused by potential insulation defects, and which might be caused by electromagnetic noise or imperfections of the measurement apparatus. Similarities between the signals are blurred by noise. Therefore, they deviate slightly from each other. Furthermore, possible reflections from defects are superposed by reflections from branching points and interconnections within the coils, making the analysis even more ambiguous.

Fast-Fourier-transform (FFT) and wavelet calculations are performed for the sake of localization of possible defects. Wavelets have the property, that an increased time resolution will decrease the spectral resolution of the analysis. Insulation defects, applied intentionally on sample cables at the distance $\Delta \mathrm{x}$ from the pulse feeding point, show in some cases a minimum of spectral energy just after the time delay of $2 * \Delta \mathrm{x} / \mathrm{V}_{\text {group. The time }}$ delay of that minimum could therefore be used as indicator for the localization of the defect. Until today, however, we had no opportunity to test this method on W7-X, because no weak insulation points close to the pulse feeding points could be detected during the last few months (fortunately).

Another technique makes use of 2D Fast-Fourier Transform (FFT) and Autoregressive Models (AR) [12] to detect possible wave propagation patterns along the wires. For that sake, the HV pulses are applied to a finite number of quench detection wires. The HV pulses will propagate along the cable, but will also be reflected at weak insulation points (but of course also on cable branches, discontinuities of the impedance etc.) such that the entire coil compound represents an oscillating system with damping, ringing, cross talk etc. As the numbers of accessible wires is limited to rather low numbers (typically 4 -64), the 2D FFT will suffer from convolution effects induced by that truncating. In the best case, we can substitute the truncating by appropriate windowing, slightly mitigating that artefact. In addition, any FFT will presume either infinite signals or at least periodicity, a condition that is here not given. Accordingly, the FFT and wavelet analyses obviously reveal in first order electronic ringing, which is excited by the HV-pulse and decays afterwards. Probably, the ringing frequency and the amplitude decay time might depend on weak insulation points (among many other parameters), and systematic investigations are planned for the future.

To overcome the drawbacks of FFT, a Burg's AR model [13] spectral estimate algorithm is used. The figures 27 and 28 show an example of the HV pulse input into 64 quench detection wires. The input and the reflected voltages are shown in fig. 27 as a function of the number of the wire (y-axis) and time (x-axis). One can see the start of the pulse at about $0.1 \times 10^{5} \mathrm{~ns}$ and the first few oscillations on the left, which look identical for all wires. The sampling rate is $1 \mathrm{GHz}$.

The calculated result for the spectral power estimate output is shown in fig. 28. This could be interpreted as the $\omega(k)$ relation for the spectral co-ordinates of the time-domain (frequency) and space (geometric spacing of the wires, $k$-wavenumber). Note that the spatial co-ordinate is unevenly spaced and not really a geometrical coordinate, but the subsequent number of wires going to the superconductor. This very indirect approach is a consequence of the impossible direct access to the coils, born from necessity. 


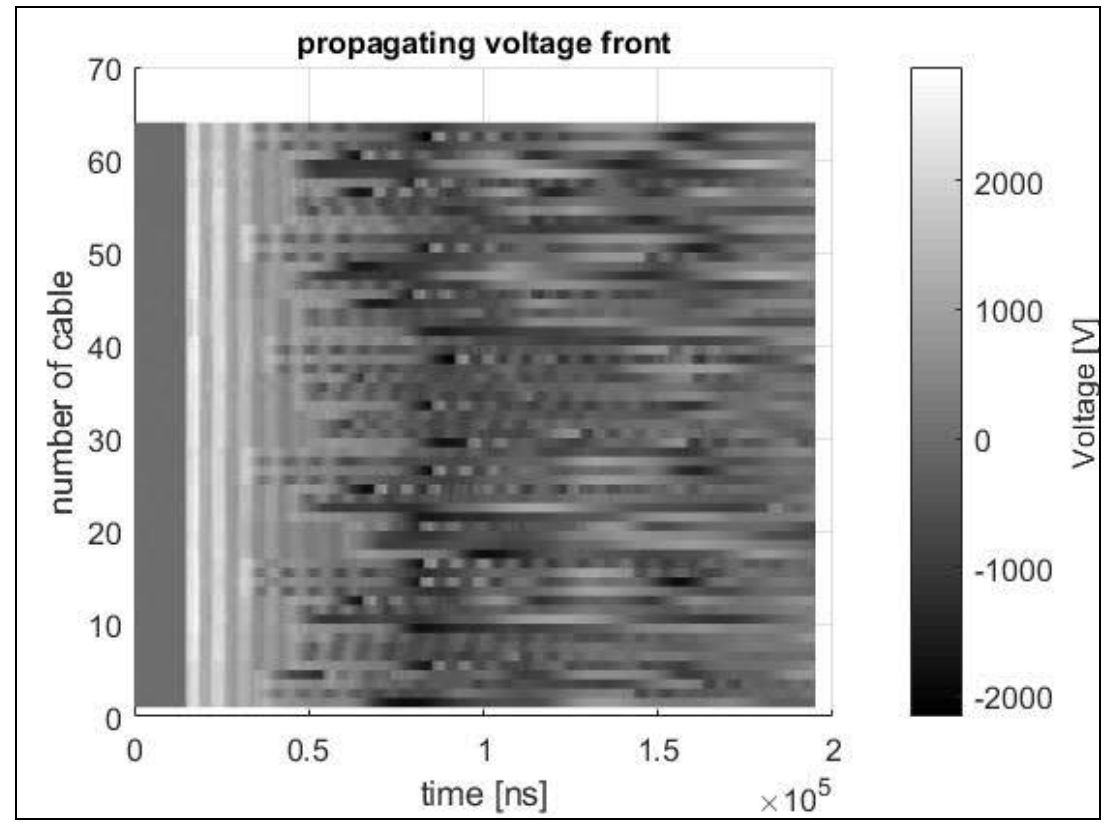

Fig. 27: Raw data of the HV pulse input to the coil system. The x-axis shows the time traces of the reflected signals, the y-axis is along the number of wires. The side bar indicates the voltage. The smooth grey area on the left hand side is the time interval prior to the HV-pulse. The first few voltage oscillations are clearly visible, then the time traces start to deviate from each other.

Obviously, the spectral estimate shows no running or sharp patterns; despite we suspected on that series of coils a defect in the insulation. During one first measurement a very slow increase in the test current was observed, developing on the time scale of several seconds, but this could fortunately not be confirmed by later measurements. In that sense, the shown pattern is the fingerprint of an intact insulation, at least up to the applied test voltage.

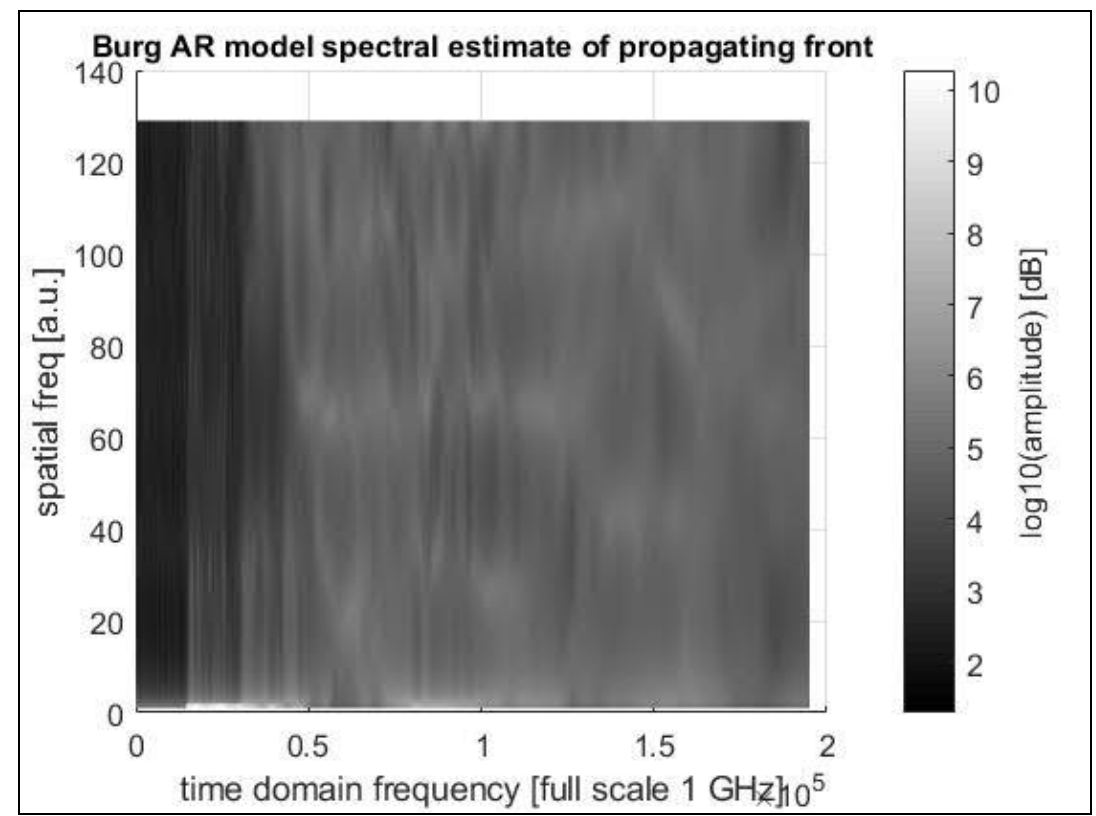

Fig. 28: Burg's AR model spectral power estimate calculation of order 6 . The time domain frequency is along the $\mathrm{x}$-axis, the full scale corresponds to $1 \mathrm{GHz}$. The y-axis is the spatial "frequency", i.e. the spectral "co-ordinate" along the number of wires. The side bar indicates the spectral estimated power in $\mathrm{dB}$. No distinct pattern can be seen, that could be indicating an obviously intact insulation.

We are aware of the fact, that the numerical methods described here probably cannot reflect appropriately the complexity of the system under consideration. Any straightforward interpretation of any result has to be taken 
with extreme caution. Obviously unambiguous findings require always confirmation by hardware tests. However, we tried to summarize here at least some of the aspects of the numerous restrictions, and we have to keep them always in mind. Any effort in understanding the test results or the outcome of numerical procedures will prevail the potential effort for more HV tests or hardware modifications on the W7-X cryostat.

The above-described techniques work in the test tank in the laboratory. But in some cases they turned out as not really suitable for the application on the W7-X coils because of the inevitable sources of noise, the very difficult accessibility, or because results from the laboratory could simply not be reproduced on W7-X because of numerous reasons. Much more activity will therefore be required to transfer the results from the laboratory to the superconductor system, in order to improve the present sample tests one day to a reliable working tool. Moreover, much more hardware modifications will be required to install the above-mentioned optical system to the W7-X cryostat, however always with the risk of damaging existing installations. Careful risk assessment for the different strategies are therefore mandatory.

\section{CONCLUSIONS}

The stellarator W7-X consists of an extremely large and complex superconducting coil system that requires permanent survey and quality control. However, no standard techniques or simple procedures exist to maintain tightness against current leaks, or to guarantee the safety against discharges in the Paschen regime. Access to the superconductor is difficult or even impossible, such that a variety of indirect measures had to be invented to maintain safe operation.

High voltage tests of the coils, or the series of coils, are performed on a regular basis. These turned out as a reliable and direct means to detect weak insulation points, in particular during the period of coil production. Paschen tests at various background pressures are also a reliable means of testing, however with the severe restriction that no helium can be used as background gas because of interference with the regular vacuum leak tests on the cryostat, and because no cryogenic tests can be done because of the enhanced heat conduction between the environment and the coils.

Low-voltage impedance tests turned out as a very powerful means to detect short-circuits and current leaks to ground, however only for the case that hard short-circuits without a gas discharge exist. Numerical simulations are mandatory to understand the observed results. Major advantage of these methods is the fact, that no stress is imposed to the insulation by the application of a high-voltage.

Run-time methods are sometimes complicated to perform, in particular if steps or branches in the cablings exist. The amount of measurement data can be huge because sampling rates in the range of $\mathrm{GHz}$ are required, and a large number of measurement points and input channels is required. The results are ambiguous and difficult to interpret; only sophisticated numerical methods can help to evaluate insulation faults. So far, it is difficult to localize defects unambiguously. As no severe high voltage faults were detected on W7-X, so far and within the specified boundaries and safety margins, no experience could be gained in testing the numerical methods and verifying their relevance for W7-X. In that case, supplementary optical methods have to be used (see above). If a single qd-wire has to be tested, however, weak insulation points could successfully be localized and repaired afterwards.

A broadband antenna system is permanently installed on W7-X. It surveys the superconductor during operation. That system turned out as rather sensitive to electromagnetic perturbations. This provides, on the other hand, confidence that even low-power sparks could be detected during tests. The possibility of performing regular and robust tests of the functionality of the system, by using a network analyzer, strengthen the confidence. During several months of operation, no event could be recorded that was unequivocally induced by an insulation defect in the superconductor.

Partial discharge measurements are performed on a regular basis on W7-X, as well, but provided so far no evidence for weak insulation points. It is impossible to say presently whether the method is sensitive enough in our case and as a matter of principle, or not. Advantages are the absence of high-voltage stress on the insulation and the rich amount of experience with PD measurements, in general.

Optical methods, either single channel detectors or imaging systems, are promising for the case that a weak insulation point had been detected with one of the methods described above. However, their localization is problematic. The tested detectors are sensitive enough to indicate even weakest sparks, and provide the advantage of remote installation in a well-shielded environment away from W7-X, provided optical access to the coil system is given and an optical fiber connection can be installed. Such an access will be opened only in the case of a real current leak, because that will mean a local destruction of the radiation shield, together with an elevated technical risk for $\mathrm{W} 7-\mathrm{X}$, high costs and a considerable expenditure of time.

\section{ACKNOWLEDGEMENT}

This work has been carried out within the framework of the EUROfusion Consortium and has received funding from the Euratom research and training program 2014-2018 and 2019-2020 under grant agreement No 633053. The views and opinions expressed herein do not necessarily reflect those of the European Commision. 
We highly acknowledge the support by the Vienna University of Technology, Atominstitut, Austria, provided during the construction and first operation phase of the superconductors.

Special thanks go to the Forschungs- und Transferzentrum Leipzig e.V., Germany, for supporting us with hardware and their personal skills for the partial discharge measurements.

Gratefully acknowledged is the support by the Fachkommission fuer Hochspannungsfragen FKH, Zuerich, Switzerland, for numerous discussions concerning high-voltage measurement techniques.

Finally we thank the Karlsruher Institut fuer Technologie, KIT Karlsruhe, Germany, for the development of hardware and the execution of Paschen-tests.

\section{REFERENCES}

[1] C. Beidler et al.,, „Physics and engineering design for Wendelstein 7-X“, Fusion Technol., issue 1, vol. 17 Jan. (1990) p. 148

[2] G. Grieger, W. Lotz, P. Merkel, J. Nührenberg, J. Sapper, H. Wobig, „Physics optimization of stellarators“, Phys. Fluids B, vol. 4 (1992) p. 2081

[3] N. Mitchell, A. Devred, P. Libeyre, B. Lim, F. Savary, the ITER Magnet Division, "The ITER magnets, design and contruction", IEEE Trans. Appl. Superconductivity, vol. 22, no. 3, June (2012), 4200809

[4] T. Rummel, F. Fuellenbach, T. Moennich, „Power supplies for the Wendelstein 7-X stellarator”, Fusion Eng. Design, vol. 66-68, Sept. (2003), p. 1115

[5] J. Baldzuhn, H. Ehmler, A. Hoelting, K. Hertel, C. Sborchia, L. Genini, T. Schild, "Coil tests and superconductor code calculations for the W7-X stellarator coils", Cryogenics, vol. 46 (2006), p. 507

[6] J. Baldzuhn, H. Ehmler, A. Hoelting, C. Sborchia, K. Hertel, L. Genini, T. Schild, "Recent results of the cold tests performed on the stellarator W7-X coils, IEEE Trans. Appl. Superconductivity, vol. 16, no. 2, June (2006), p. 763

[7] M. Schoenhuber, "Breakdown of gases below Paschen minimum”, IEEE Trans. Power Apparatus Systems, vol. 88, no. 2, Feb. (1968), p. 100

[8] W. Hayt, Engineering Electromagnetics, Mc Graw-Hill. (5 ${ }^{\text {th }}$ Edition) (1989)

[9] F. Auzanneau, "Wire troubleshooting and diagnosis: review and perspectives", Progress in Electromagnetics Research B, vol. 49 (2013) p. 253

[10] https://www.cfx-berlin.de/software/elektromagnetik/simplorer.html, "Engineering Simulation \& 3D Design Software" and https://www.ansys.com/ (2019)

[11] A. Kuechler, Hochspannungstechnik, Springer-Verlag, Berlin, Heidelberg. (3 ${ }^{\text {rd }}$ Edition) (2009)

[12] E. Parzen: Handbook of Statistics, Time Series in the Frequency Domain, Vol. 3 (1983), Elsevier B.V.

[13] J.P. Burg: Modern Spectrum Analysis, Ed. D.G. Childers, New York IEEE Press. (1978) p. 42

\section{E-mail of the author: baldzuhn@ipp.mpg.de}

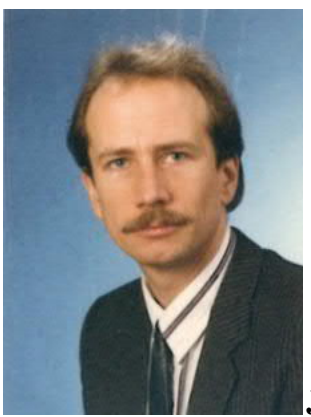

J. Baldzuhn received his Diploma Thesis in the field of Fourier-Transform Infrared Spectroscopy (FTIR) for bio-medical investigations in the field of signal theory from the Albert-Ludwigs University, Freiburg i. Br., Germany in 1986. Focus was on Maximum-Entropy methods and hardware of FTIR spectrometers. He received his Ph.D. degree Dr. phil. nat. in quantum optics from the Goethe University, Frankfurt a.M., Germany in 1990. Major subjects were laser optics and delayed-choice experiments with single photon states in order to investigate the collapse of quantum states in various space-time domains, and to highlight the non-objectivity of quantum mechanical systems. Since 1990 he is researcher at the Max-Planck Institute for Plasma Physics, Germany. His activities are the charge exchange spectroscopy of plasma impurities within neutral beams in order to measure electric and magnetic fields inside the plasma, and ion temperatures. Numerical neoclassical code calculations support the measurements and help to illuminate transport phenomena of energy and particles in a fusion relevant magnetized plasma. He is also responsible for the injection of cryogenic hydrogen ice pellets, for the purpose of deep particle fueling. During the installation of the stellarator Wendelstein 7-X in Greifswald, Germany, he was responsible for the cryogenic and high-voltage tests of the superconducting coils. 


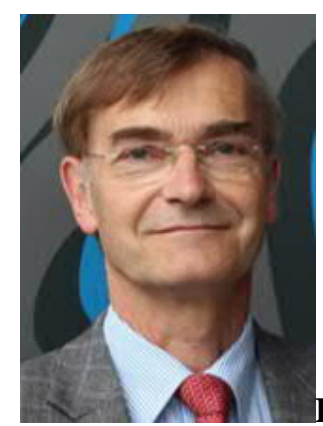

H. S. Bosch has studied physics in Münster and Munich. He received the Dr. rer. nat. degree from the Technical University, Munich, in 1986, and the Habilitation degree from Humboldt University, Berlin, Germany, in 2000. In August 2016 he was appointed as a professor at TU Berlin.

After his post-doc- time at TFTR in Princeton, he worked on ASDEX and ASDEX Upgrade at Max-PlanckInstitute for Plasma Physics, Garching. In 2000 he changed the field, becoming head of the Director's Office. In 2004 he joined the project Wendelstein 7-X as head of Project Coordination. Since 2013 he is the "Director Operations" for Wendelstein 7-X and deputy head of W7-X. In this function he has led the first three operation phases and the corresponding commissioning phases.

K. Gallowski photograph and biography not available at the time of publication.

H. Grote photograph and biography not available at the time of publication.

T. Klinger photograph and biography not available at the time of publication.

C. Kopplin photograph and biography not available at the time of publication.

M. Nagel photograph and biography not available at the time of publication.

K. Rummel photograph and biography not available at the time of publication.

T. Rummel photograph and biography not available at the time of publication.

K. Risse photograph and biography not available at the time of publication.

F. Schauer photograph and biography not available at the time of publication.

O. Volzke photograph and biography not available at the time of publication.

L. Wegener photograph and biography not available at the time of publication.

M. Weise photograph and biography not available at the time of publication.

Andreas Werner photograph and biography not available at the time of publication. 